\title{
From UBGs to CFGs A Practical Corpus-Driven Approach
}

\author{
Hans-Ulrich Krieger
}

August 2004

Deutsches Forschungszentrum für Künstliche Intelliaenz GmbH

Postfach 2080

67608 Kaiserslautern, FRG

Tel.: + 49 (631) 205-3211

Fax: + 49 (631) 205-3210

E-Mail: info@dfki.uni-kl.de
Stuhlsatzenhausweg 3

66123 Saarbrücken, FRG

Tel.: + 49 (681) 302-5252

Fax: + 49 (681) 302-5341

E-Mail: info@dfki.de

WWW: http://www.dfki.de 



\section{Deutsches Forschungszentrum für Künstliche Intelligenz DFKI GmbH German Research Center for Artificial Intelligence}

Founded in 1988, DFKI today is one of the largest nonprofit contract research institutes in the field of innovative software technology based on Artificial Intelligence (AI) methods. DFKI is focusing on the complete cycle of innovation - from world-class basic research and technology development through leading-edge demonstrators and prototypes to product functions and commercialization.

Based in Kaiserslautern and Saarbrücken, the German Research Center for Artificial Intelligence ranks among the important "Centers of Excellence" worldwide.

An important element of DFKI's mission is to move innovations as quickly as possible from the lab into the marketplace. Only by maintaining research projects at the forefront of science can DFKI have the strength to meet its technology transfer goals.

The key directors of DFKI are Prof. Wolfgang Wahlster (CEO) and Dr. Walter Olthoff (CFO).

DFKI's six research departments are directed by internationally recognized research scientists:

Image Understanding and Pattern Recognition (Director: Prof. Thomas Breuel)

$\square$ Knowledge Management (Director: Prof. A. Dengel)

Intelligent Visualization and Simulation Systems (Director: Prof. H. Hagen)

Deduction and Multiagent Systems (Director: Prof. J. Siekmann)

$\square$ Language Technology (Director: Prof. H. Uszkoreit)

口 Intelligent User Interfaces (Director: Prof. W. Wahlster)

Furthermore, since 2002 the Institute for Information Systems (IWi) (Director: Prof. AugustWilhelm Scheer) is part of the DFKI.

In this series, DFKI publishes research reports, technical memos, documents (eg. workshop proceedings), and final project reports. The aim is to make new results, ideas, and software available as quickly as possible.

Prof. Wolfgang Wahlster

Director 


\section{From UBGs to CFGs \\ A Practical Corpus-Driven Approach}

Hans-Ulrich Krieger

DFKI-RR-04-01 
First of all, my thanks go to Bernd Kiefer for providing me with the modified parsers and for helping me adapting the grammars. His help during the measurements is gratefully acknowledged. I have also benefitted from many discussions with him. Thanks to Uli Callmeier for making flop and PET available to the public. I am grateful to Feiyu Xu for making comments on a preliminary version of this paper. Finally, I like to thank the three ICGI reviewers for their detailed and encouraging comments.

This work was supported in a first phase by the Verbmobil project (grant no. 01 IV 701 V0), resulting in a LISP implementation of the approach in summer 1999, realized on top of the Verbmobil deep core engine (Kiefer et al. 2000). This first implementation of the extraction method was used by (Nederhof 2000) to produce CFGs of different size and specificity during his experiments with regular approximation of context-free languages. An extended first JAVA reimplementation was finished in autumn 2002, was further extended in spring 2004, and will be employed shortly in the QUETAL project. Parts of the implementation, the writing of this report, and the measurements were funded by the German BMBF under grant no. 01 IW C02 (QUETAL).

\section{(C) Deutsches Forschungszentrum für Künstliche Intelligenz 2005}

This work may not be copied or reproduced in whole or part for any commercial purpose. Permission to copy in whole or part without payment of fee is granted for nonprofit educational and research purposes provided that all such whole or partial copies include the following: a notice that such copying is by permission of the Deutsche Forschungszentrum für Künstliche Intelligenz, Kaiserslautern, Federal Republic of Germany; an acknowledgement of the authors and individual contributors to the work; all applicable portions of this copyright notice. Copying, reproducing, or republishing for any other purpose shall require a licence with payment of fee to Deutsches Forschungszentrum für Künstliche Intelligenz. 


\title{
From UBGs to CFGs A Practical Corpus-Driven Approach
}

\author{
Hans-Ulrich Krieger \\ German Research Center for Artificial Intelligence (DFKI) \\ Language Technology Lab \\ Stuhlsatzenhausweg 3, D-66123 Saarbrücken, Germany \\ krieger@dfki.de
}

August 2004

\begin{abstract}
We present a simple and intuitive unsound corpus-driven approximation method for turning unification-based grammars (UBGs), such as HPSG, CLE, or PATR-II into context-free grammars (CFGs). The method is unsound in that it does not generate a CFG whose language is a true superset of the language accepted by the original unification-based grammar. It is a corpus-driven method in that it relies on a corpus of parsed sentences and generates broader CFGs when given more input samples. Our open approach can be fine-tuned in different directions, allowing us to monotonically come close to the original parse trees by shifting more information into the context-free symbols. The approach has been fully implemented in JAVA. This report updates and extends the paper presented at the International Colloquium on Grammatical Inference (ICGI 2004) and presents further measurements.
\end{abstract}




\section{Introduction}

We present a simple and intuitive unsound corpus-driven approximation method for turning unification-based grammars (UBGs), such as HPSG (Pollard and Sag 1994), CLE (Alshawi 1992), or PATR-II (Shieber et al. 1983), into context-free grammars (CFGs). The method is unsound (Pereira and Wright 1991, p. 246) in that it does not generate a CFG whose language is a true superset of the language accepted by the original unification-based grammar. It is a corpus-driven method in that it relies on a corpus of parsed sentences and generates broader CFGs when given more input samples. Our open approach can be fine-tuned in different directions, allowing us to monotonically come close to the original parse trees by shifting more information into the context-free symbols. The approach has been fully implemented in JAVA. This report updates and extends the paper presented at the International Colloquium on Grammatical Inference (ICGI 2004, Krieger 2004a) and presents further measurements.

\subsection{Motivation}

The motivation for developing this corpus-based approximation method was manifold.

Two-Step Parsing. An approximated CFG can be seen as a cheap recognition pre-filter during NL parsing with a worst-case running time of $O\left(n^{3}\right)$ for a sentence of length $n$, whereas UBG parsing usually comes up with an exponential worstcase complexity. Given the existence of such a pre-filter, a unification-based parser thus only needs to deterministically replay the CF parse trees afterwards, i.e., must not fully explore its search space during all-path parsing (Kiefer and Krieger 2002, Kiefer and Krieger 2004). UBG tree reconstruction is easily established by a total many-to-one mapping (a surjective function) from CFG rules onto UBG rules.

Disambiguation of UBGs. By moving from the approximated CFG and a training corpus to a PCFG that predicts probabilities for $\mathrm{CF}$ parse trees, we also obtain an indirect and efficient stochastic parsing model for the UBG, even though the UBG might encode an infinite number of categories. I.e., we shift the responsibility for predicting the 'right' derivation trees to the context-free side, where mathematically clean methods exist. Using the probabilities, we can dynamically rank the CF parse trees at runtime, and again, let the UBG parser deterministically replay these trees. This establishes an order on the UBG trees that can be employed for UBG disambiguation (Kiefer et al. 2002).

Domain-Specific Subgrammars. The extraction of useful domain-specific context-free subgrammars from general-purpose unification-based grammars is an important topic in information extraction and question answering (Neumann 2003). Our approach thus may obviate the laboriously manual construction of such domain-specific grammars. As we will see later, our method neither produces a superset nor a subset of the language generated by the original UBG. However, this is a desired effect, since a domain-specific subgrammar is not intended to generate a true superset: it should cover the relevant constructions of 
the domain and only these constructions (in the best case). And because it is also not a true subset of the UBG, it can be robust against ungrammatical input w.r.t. the domain we are interested in.

Context-Free Language Models. Given an UBG, it would be nice to have an automated compilation method that yields a CFG which in turn serves as a symbolic, word-based (instead of phoneme-based) context-free language model, guiding a speech recognizer (Rayner et al. 2001a). This strategy obviates the sparse data problem in (commercial) recognizers, since we can directly operate on the high-level grammar without collecting and constructing large amounts of annotated speech training material (Wizard of $\mathrm{Oz}$ ). The automatic compilation of a CFG from an UBG also makes a tedious hand-coded formulation of a CFG (or a regular grammar) superfluous (Dowding et al. 2001). Findings in Rayner et al. 2001b suggest that agreement constraints in context-free language models improve the performance of a recognizer in terms of both word error rate and semantic error rate. Keeping the agreement constraints of the UBG in the CFG is easy in our approximation method. Finally, CF-based models clearly benefit from their greater expressive power when compared to regular models. It is worth noting that within the last four years or so, the commercial speech community has focused primarily on the grammar-based approach (VoiceXML, W3C, Nuance, Speech Works, etc.); see also Rayner et al. 2001b.

Open Approach. The adjusting parameters of our approach make it easy to approximate CFGs of different size and quality, e.g., by varying the annotations of context-free symbols, by collecting the approximated CF rules either under rule equivalence or under rule subsumption, or by taking a larger domain of locality into account, by using the feature structures of the UBG, resulting in various forms of context-free tree grammars. Even though these tree grammars are still context-free, they clearly have a larger event horizon, establishing a restricted form of look-ahead.

\subsection{General Idea}

Since unification-based parsers usually rely on a context-free backbone of unification rules (or rule schemata, to borrow the broader HPSG term), it should not be that difficult to extract a context-free grammar. In fact, relatively specific unification-based rules (e.g., ANLT, Carroll et al. 1991 or LFG, Kaplan and Bresnan 1982), should result in approximated CFGs of good quality (cf. Carroll 1993; see section 4 for a discussion of other approaches). However, lexicalized grammar theories such as HPSG, or even categorial grammar frameworks, like CUG (Uszkoreit 1986) or UCG (Zeevat et al. 1987), are of a different kind: rule schemata in these frameworks are usually so general that the resulting CFGs are worthless, meaning that they accept nearly everything (Briscoe and Carroll 1993, p. 36). Proper recognition of utterances in lexicalized theories is realized by shifting the great amount of information into the lexicon and by applying a specific descriptive means in rule schemata: coreferences or reentrancies.

Our attempt thus does not operate on the rules of a unification-based grammar (as do, e.g., Kiefer and Krieger 2002 in their sound HPSG approximation), but 
instead on valid rule instantiations of a special kind, viz., passive edges of the unification chart, resulting from parsing a corpus. In order to access such passive edges, we have defined an external exchange format for representing a chart (see section 3.1). Since passive edges directly encode their immediate daughters, a passive edge can be seen as a tree of depth 1 . From such a tree and with the help of the feature structure directly associated with each passive edge, it is possible to create an annotated context-free rule of arbitrary specificity (section 3 ). Terminal and nonterminal symbols in our framework are equipped with information from the related feature structure of the passive edge, similar to annotated symbols in the GPSG framework (Gazdar et al. 1985; see section 2.2). When taking deeper nested daughters into account, we can even escape the flat domain of contextfree rules, resulting in CF tree grammars (see Neumann 2003 for a tree-based approach resulting from a treebank).

In order to predict probabilities for CF parse trees, we equip each rule with a frequency counter which tells us how often a rule has been successfully applied when parsing a training corpus. Given these counters, it is then easy to move from the extracted CFG to a trained PCFG (Lari and Young 1990) which might be employed during parsing in order to disambiguate context-free readings. Assuming that the extracted $\mathrm{CFG}$ does not produce too many additional readings for the relevant syntactic constructions in the corpus when compared to the UBG, the PCFG can thus be seen as an indirect probability model for the UBG. The trick goes as follows. Since every CFG rule is related by its rule name to a unification rule, we first let the PCFG parse a given input, predicting probabilities for CF parse trees. In a second phase, the ranked parsing trees can be deterministically replayed one after another by the UBG (processing the most probable CFG trees first), establishing an order of best UBG parsing trees (see Kiefer et al. 2002 for first promising results). Clearly, this idea only works for utterances lying in the intersection of the languages accepted by the UBG and the approximated CFG. Independently of establishing a stochastic parsing model, the two-step parsing process alone can be used to speed up unification-based (all-path) parsing by employing the same above idea (as has been proposed by many groups, most notably, the LFG community): only the predicted context-free derivation trees are deterministically reparsed by the UBG, helping the unification-based parser to reduce its search space. Kiefer and Krieger 2002 have shown that two-stage parsing is feasible, even with large approximated CF grammars of more than 600,000 to $1,500,000$ rules, resulting in a speedup of $41 \%-62 \%$.

Since the form and size of an approximated CFG is largely determined by the training corpus (contrary to the pure grammar-driven approach in Kiefer and Krieger 2002 and Kiefer and Krieger 2004), our approach makes it easy to compute domain-specific subgrammars from general large-scale unification grammars. Thus this approach might gain importance in information extraction and related tasks. In other words, the syntax of a domain is addressed by the linguistic constructions in the training corpus, whereas the reconstruction of the proper domain-specific semantics is realized by manually (or semi-automatically) selecting the 'right' UBG parse trees, before approximating the CFG. I.e., the approximation procedure will not see the 'wrong' UBG parse trees and so, 'wrong' CF rules will not appear in the final CFG. 


\subsection{Structure of Report}

The structure of this report is as follows. In the next section, we first introduce some basic inventory (types, type hierarchy, typed feature structures, unification) and discuss the objects which are constructed and manipulated during the extraction of the CFG (symbols, rules, edges). After that, section 3 presents the interface to the chart of the HPSG parser and describes the basic extraction algorithm, together with a variation which produces smaller, although more general CFGs. The section also has a few words on the quick-check paths of the UBG which serve as the starting point for finding the proper annotations of the context-free symbols. Section 4 then comes with a discussion of other approaches which aim at extracting a CFG from an UBG, most of them unsound. In section 5 , we elaborate on further aspects of the extraction methods, discussing several orthogonal adjusting parameters which result in different CFGs, given a UBG. We also discuss additional postprocessing steps and motivate that the extracted CFGs can be tuned to deliver a meaningful semantic output as well. Finally, in section 6, we apply our method to several small- to large-size UBGs and present first measurements for the large English Resource Grammar developed at CSLI, Stanford.

\section{Objects of Interest}

The goal of this section is a description of the implemented objects which are built and manipulated by the extraction algorithm (section 3 ). The section furthermore defines certain important relations between symbols and rules. It also has a few introductory remarks concerning typed feature structures.

\subsection{Typed Feature Structures}

This subsection introduces some fundamental theoretical concepts which are used throughout the paper. A more thorough investigation can be found in, e.g., Shieber 1986, Carpenter 1992, and Krieger 1995.

Definition 1 (TYPE HIERARCHY)

Let $\mathcal{T}$ be a finite set of type symbols. We refer to a type hierarchy by a pair $\langle\mathcal{T}, \preceq\rangle$, such that $\preceq \subseteq \mathcal{T} \times \mathcal{T}$ is a decidable partial order (Krieger 2001). I.e., $\preceq$ is

- reflexive: $\forall t \in \mathcal{T}$. $t \preceq t$

- antisymmetric: $\forall t_{1}, t_{2} \in \mathcal{T} \cdot t_{1} \preceq t_{2} \wedge t_{2} \preceq t_{1} \Rightarrow t_{1}=t_{2}$

- transitive: $\forall t_{1}, t_{2}, t_{3} \in \mathcal{T} . t_{1} \preceq t_{2} \wedge t_{2} \preceq t_{3} \Rightarrow t_{1} \preceq t_{3}$

In the following, we assume that $\mathcal{T}$ contains three special symbols: $T$ (the most general type), $\perp$ (the most specific type), and $\mathrm{U}$ (expressing undefinedness), such that $\perp \preceq t$ and $t \preceq T$, for all $t \in \mathcal{T}$. Furthermore, $\mathrm{U}$ is a direct subtype of $\mathrm{T}$ and is incompatible with every type in $\mathcal{T} \backslash\{\mathrm{T}, \mathrm{U}\}$, i.e., $\mathrm{U}$ does not have any subtype, 
except $\perp$. Wo will use the undef type $U$ later to express the fact that a cortain attribute is not appropriate for a given type.

We note here that the implementation of the corpus-driven approximation of the CFG from an UBG not only operates on a partial order of types, but even on a bounded complete partial order (or equivalently, on a lower semilattice). This is due to the fact that our JAVA implementation of typed feature structures (Krieger 2004b) takes as input a completion of the original type hierarchy, constructed from UBG by the flop preprocessor (Callmeier 2001) of the PET system (Callmeier $2000)$. Completing a type hierarchy means that for every pair of two types $t_{1}, t_{2} \in$ $\mathcal{T}$, the greatest lower bound (GLB) is defined (and there is exactly one GLB for $t_{1}$ and $t_{2}$ ). Ait-Kaci 1986 showed that every partial order can be embedded into a bounded complete partial order such that all GLBs are preserved.

Given such a completed type hierarchy, the type unification operation $\wedge$ between two types $t_{1}, t_{2} \in \mathcal{T}$ is defined to be the GLB of $t_{1}$ and $t_{2}: t_{1} \wedge t_{2}:=\operatorname{GLB}\left(t_{1}, t_{2}\right)=t$, such that $t \preceq t_{1}, t \preceq t_{2}$ and $\nexists t^{\prime} \in \mathcal{T}$ with $t \preceq t^{\prime} \preceq t_{1}$ and $t \preceq t^{\prime} \preceq t_{2}$.

We also need the notion of a typed feature structure (TFS) and will frequently talk about the finite set of features $\mathcal{F}$ (often called attributes), the possibly infinite: set of atoms $\mathcal{A}$ (often called constants), and the already mentioned finite set of types $\mathcal{T}$ (often called sorts). ' However, we will not present a definition here and only note that there exist orthogonal, although precise definitions of what TFSs are (the enumeration is, of course, not complete):

- a kind of deterministic finite state automaton (Carpenter 1992)

- an extension of Ait-Kaci's $\psi$ terms (Kricger 1995)

- syntactic sugar/expressions in a designer logic ${ }^{2}$ which can bo transformed into definite equivalences (Krieger 2001)

- elements of the least solution of a certain recursive domain exuation (Kiefer and Krieger 2002)

From an implementation point of view, TFSs are not that different from records (structures) in imperative programming languages (e.g., PAScAL, C) or classes in object-oriented languages such as JAVA or $\mathrm{C}++$. They can also be seen as a generalization of unnamed tuples and fixed-arity terms (as, e.g., in Prolo(i). Given a TFS, a feature expresses a functional property (i.e., having exactly one value) and its value might again be a highly-structured TFS. This allows the construction of deep-nested, arbitrary-complex objects and in fact, the TFSs delivered by the HPSG parser are of that kind.

TFSs also possess another interesting descriptive means, viz., coreferences or rocntrancies. They help to state the fact that the values under at least two features within a TFS are identical (and not merely structural equal). Coreferences thus enforce agreement and furthermore are a means for information transport during the unification of two TFSs. In this setting here, viz., UBG parsing, unification

\footnotetext{
${ }^{1}$ Many typed feature-based systems do not distinguish between $\mathcal{A}$ and $\mathcal{T}$ and thus must explicitly enumerate such atomic types.

${ }^{2}$ A term coined by Mark Johnson.
} 
is merely employed for checking satisfiability during rule instantiation and for building up (output) structure.

We close this subsection by defining the notion of a path. Putting it simply, a path is a sequence of features $f_{1}, f_{2}, \ldots, f_{n} \in \mathcal{F}$ which helps us to access information from deeper levels of a TFS. We depict such a path as $f_{1}\left|f_{2}\right| \ldots \mid f_{n}$. One specific path stands out, viz., the empty path $\epsilon$, referring to the TFS itself.

\subsection{Symbols}

Terminal and nonterminal symbols of the context-free grammar are represented as instances of the JAVA class Symbol. ${ }^{3}$ Symbols bear a name field of type String and an annotation field of type int [] (an integer array). The name field of a terminal refers to the full surface string of this terminal word and its annotation field is empty (refers to the null value). A nonterminal also has a name and encodes the HPSG rule name (e.g., hcomp or measure_np). The annotation of a nonterminal symbol groups several type identifiers which originate from (possibly deep-nested) values under pre-specified paths (the so-called annotation paths) within the instantiated rule TFS for this nonterminal symbol. Thus an annotation is quite similar to a feature specification in GPSG (Gazdar et al. 1985) or a quickcheck vector (Kiefer et al. 1999, Malouf et al. 2000).

During the more formal parts of this paper, we need two fundamental concepts: symbol and annotation.

Definition 2 (SYMBOL)

We refer to a symbol $s$ in the abstract syntax by a pair $\langle n, a\rangle$, consisting of name $n$ and an annotation $a$. We write $N(s)$ to depict the first projection of $s$ (the name) and $A(s)$ to access the annotation part of $s$. When using concrete syntax, we write a symbol in the more GPSGish notation $\mathrm{n}[\mathrm{a}]$.

\section{Definition 3 (ANNOTATION)}

An annotation $a=\left\langle t_{1}, \ldots, t_{n}\right\rangle$ is a $n$-tuple of type names or type IDs $t_{i} \in \mathcal{T}$, $i \in\{1, \ldots, n\}$. We write $\pi_{i}(a)$ to denote value $t_{i}$ of the $i$-th projection of $a$. Using the GPSG-like concrete syntax, we write $\left[t_{1}, \ldots, t_{n}\right]$ to depict annotation $a$.

Given two symbols, we define a subsumption relation $\preceq$ which turns out to be useful in a moment.

\section{Definition 4 (SYMBOL SUBSUMPTION)}

Let $s_{1}$ and $s_{2}$ be two annotated context-free symbols. $s_{1}$ is said to be subsumed by $s_{2}$ (written as $\left.s_{1} \preceq s_{2}\right)$ iff $N\left(s_{1}\right)=N\left(s_{2}\right)$ and $\pi_{i}\left(A\left(s_{1}\right)\right) \preceq \pi_{i}\left(A\left(s_{2}\right)\right)$, for all $i \in\{1, \ldots, n\}$. Alternatively, we say that $s_{2}$ subsumes (or is more general than) $s_{1}$. Assuming that $s_{1}$ and $s_{2}$ are terminal symbols, their annotation must be empty $\left(A\left(s_{1}\right)=A\left(s_{2}\right)=\langle\rangle\right)$, thus the second condition above is trivially satisfied. ${ }^{4}$

\footnotetext{
${ }^{3}$ Note that during the more technical aspects of this paper, I will adopt the JAVA notation and its specific language use, e.g., by referring to an instance variable as a field. See, e.g., Flanagan 2002.

${ }^{4}$ Note that we have overloaded (and in the following will further overload) the $\preceq$ relation.
} 
Let us give an example. Assume we have the four CF symbols (concrete syntax) $N[s g, f e m], N[p l, f e m], N[n u m, f e m], D[n u m, f e m]$ and assume that $s g \preceq$ num and $\mathrm{pl} \preceq$ num is the case. We can then infer that $\mathrm{N}[\mathrm{sg}, \mathrm{fem}] \preceq \mathrm{N}[\mathrm{num}, \mathrm{fem}]$ and $\mathrm{N}[\mathrm{pl}, \mathrm{fem}] \preceq \mathrm{N}[\mathrm{num}, \mathrm{fem}]$ holds. However, $\mathrm{N}[\mathrm{sg}, \mathrm{fem}]$ and $\mathrm{N}[\mathrm{pl}$, fem] are not related by the subsumption relation. Consequently, we say that these symbols are incompatible (or incomparable) and use the $\bowtie$ sign to indicate this: $\mathrm{N}$ [sg,fem] $\bowtie$ $\mathrm{N}[\mathrm{pl}, \mathrm{fem}]$. Furthermore, $\mathrm{D}[\mathrm{num}, \mathrm{fem}]$ is incompatible with every othor symbol, due to its name: $\mathrm{D} \neq \mathrm{N}$.

\subsection{Rules}

We represent context-free grammar rules by the class Rule whose instances have a left-hand side (lhs) and a right-hand side (rhs) field. The left-hand side is (from a CFG point of view) a nonterminal symbol, which we represent as an instance of the class Symbol. The right hand-side is an array of Symbol objects (JAVA notation: Symbol []). The length of $r$ hs is encoded in an additional field length of type int. A Rule object also possesses a frequency field, telling us how often that rule has been applied during parsing of a given corpus. It is worth noting that the frequency counter will later gain importance when we we move from the cxtracted CFG to the associated PCFG which will predicts probabilities for CFG trees.

Next, we need the formal notation of a grammar rule.

Definition 5 (CONTEXT'FREE GRAMMAR RULE)

Let $l, r_{1}, \ldots r_{n}$ be (annotated) context-free symbols and let furthermore $l$ be a nonterminal symbol. We then call the expression $l \rightarrow r_{1} \ldots r_{n}$ a context-frec grammar rule. $l$ is usually referred to as the left-hand side (LHS) of the rule, whereas the sequence $r_{1} \ldots r_{n}$ is called the right-hand side (RHS). Given a CF rule $\alpha$, the projection $L$ yields the LHS of $\alpha$, i.e., $L(\alpha)=l$. $R$ delivers the RIIS: $R(\alpha)=r_{1} \ldots r_{n}$.

Given the above apparatus, defining a subsumption relation $\preceq$ on rules is relatively straightforward.

Definition 6 (RULE SUBSUMPTION)

Let $\alpha=\left(l_{\alpha} \rightarrow r_{1 \alpha} \ldots r_{n \alpha}\right)$ and $\beta=\left(l_{\beta} \rightarrow r_{1 \beta} \ldots r_{n \beta}\right)$ be two context-free grammar rules. We say that $\alpha$ is subsumed by $\beta$ and write $\alpha \preceq \beta$ iff $l_{\alpha} \preceq l_{\beta}, n_{\alpha}=n_{\beta}$, and $r_{i \alpha} \preceq r_{i \beta}$, for all $1 \leq i \leq n_{\alpha}$. Alternatively, we say that $\beta$ subsumes (or is more general than) $\alpha$.

We say that two CF rules $\alpha$ and $\beta$ are equivalent iff they both subsume each other: $\alpha \equiv \beta: \Longleftrightarrow \alpha \preceq \beta$ and $\beta \preceq \alpha$.

Let $\alpha \npreceq \beta$ abbreviate $\neg(\alpha \preceq \beta)$. Two rules are said to be incompatible (or incomparable) iff they are not related by rule subsumption: $\alpha \bowtie \beta: \Longleftrightarrow \alpha \npreceq \beta$ and $\beta \npreceq \alpha$.

An example. Given the symbols from the example at the end of subsection 2.2 , we define a subsumption order over the following three rules (we use again the concrete syntax in which the implemented algorithm delivers the rules). Let $\alpha=(\mathrm{NP}[\mathrm{sg}, \mathrm{fem}] \rightarrow \mathrm{N}[\mathrm{sg}, \mathrm{fem}]), \beta=(\mathrm{NP}[\mathrm{pl}, \mathrm{fem}] \rightarrow \mathrm{N}[\mathrm{pl}, \mathrm{fem}])$, and $\gamma=$ $(\mathrm{NP}[$ num,fem $] \rightarrow \mathrm{N}[$ num, fem] $)$. Then $\alpha \preceq \gamma$ and $\beta \preceq \gamma$, however $\alpha \bowtie \beta$. 


\subsection{Edges}

The edges which are transmitted by the HPSG parser in plain text (see figure 1) are reconstructed in main memory within the JAVA virtual machine. At the moment, we are using a modification of the freely available PET parser (Callmeier 2000). The set of all passive edges for a single parsed sentence are grouped in a text file (see next section).

Edges are represented as instances of the JAVA class Edge, consisting of the instance fields id, ruleName, immDtrs, noOfDtrs, and annotation. The id of an edge is a handle to the edge object and allows other edges to refer to this edge in their immediate daughters array immDtrs. id is of type int, thus immDtrs must be of type int []. The string in the ruleName field of the edge leads to the primary category symbol of the LHS of the context-free rule later. As already described, annotations are represented as int vectors. The extraction algorithm in the next section produces for each given edge exactly one context-free rule. Due to the ruleName field, we know the name of the UBG rule from which the edge has been derived. Exactly this information is utilized during the deterministic second phase of two-stage parsing.

\section{Extracting a Context-Free Grammar}

This section centers around the offline extraction of a context-free grammar from a given corpus, originally parsed by the deep HPSG parser of the PET system (Callmeier 2000). We first describe the textual interface between our extraction component and PET. After this, we motivate that the annotation values of context-free symbols for a recognition grammar are related to the quick-check paths, originally introduced within the context of deep HPSG parsing (Kiefer et al. 1999). Given this background, we then describe the basic extraction algorithm in pseudo code. Finally, we argue for an extension of the original algorithm, which helps to compute smaller grammars and describe how the frequencies for the context-free rules are obtained.

\subsection{Interface to HPSG}

The interface to HPSG is established via the creation of text files: for every input sentence of the corpus, a new file is created that contains exactly the passive edges produced by PET. Although not every deep passive edge contributes to a deep reading, we have decided to take all passive edges into account (one can think of other options as well; see section 5). Since the passive edges of the deep parser are objects in main memory and since our extraction runs in a separate thread, we have defined an ASCII-based exchange format for chart edges that is given by the EBNF in figure 1. Figure 2 then displays the stripped-down chart for the sentence Kim loves Sandy. Due to the fact that features and types are represented as integers in PET, it is important that both PET and the extraction process operate on the same $\mathcal{T} \mathcal{D} \mathcal{L}$ (Krieger and Schäfer 1994) grammar.

Because HPSG requires all relevant information to be contained in the synsem feature of the mother structure, the unnecessary daughters (which are part of the 


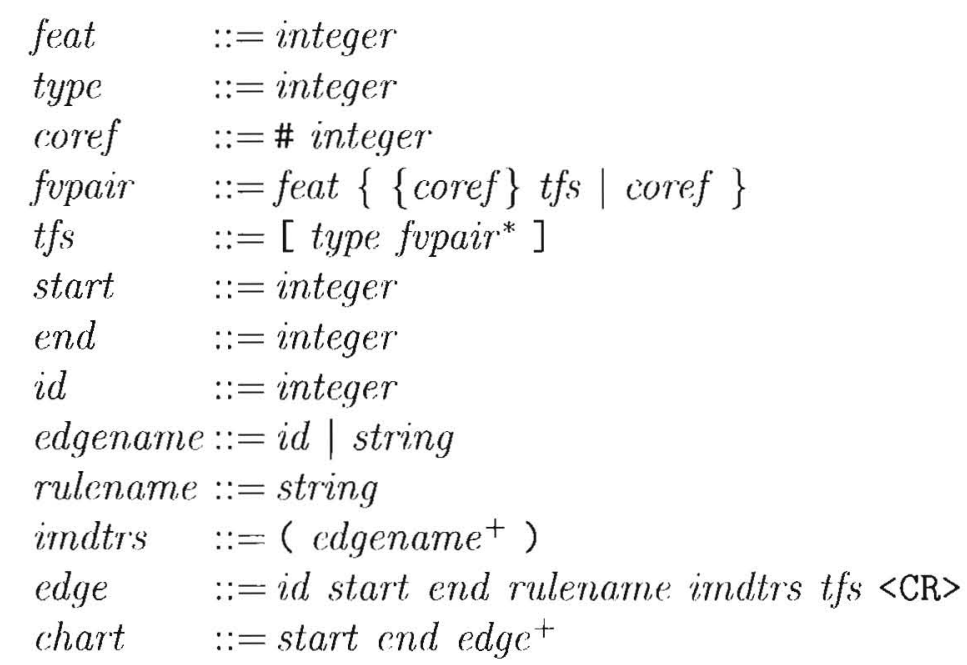

Figure 1: The external exchange format of a chart as delivered by a modified version of the PE: system. The meta characters \{ and $\}$ express optionality, | enforces the choice of exactly one alternative, and ${ }^{*}$ and + refer to Kleene star and Kleene plus, resp. integer denotes the set of all integers, i.e., sequences of numeric decimal characters. string denotes the set of all strings, i.e., sequences of plain text characters, enclosed by double quotes. Note that each chart edge must be separated by the newline character $\langle\mathrm{CR}\rangle$ at the end. At the momont, wo do not benefit from the start and end position of an edge. rulename serves as the main category symbol. imdtrs refers to the immediate daughters of an edge. Since the delivered passive edges are topologically sorted, it is not necessary to reencode an edge that has already been introduced carlier. Thus the immediate danghters imdtrs of an edge are referred to by integer numbers id. The only exception are terminal symbols (the surface form) which are written as pure strings.

TFS) only increase the size of the overall feature structure without constraining the search space. Due to the Locality Principle of HPSG (Pollard and Sag 1987, pp. 145), they can therefore be legally romoved in fully instantiated items, i.e., passive edges which are delivered by the PET parser. To be independent from a cortain grammatical theory or implementation, we use restrictors similar to Shieber 1985 as a flexible and easy-to-use specification to perform this deletion. In case we are trying to work with a larger tree context and not limiting ourselves to context-free rules (= trees of depth 1 ), the restrictor is the right means to accomplish this (see section 5).

\subsection{Quick-Check Paths}

Quick-check paths are used during unification-based parsing to quickly and correctly filter out failing unifications without applying the more costly unification operation (Kiefer et al. 1999, Malouf et al. 2000). Such a filter is extremely important since most of the unifications usually fail (95-99\% of all unifications). The quick-check filter exploits the fact that unification fails more often at certain points in feature structures than at others. In order to determine the most 


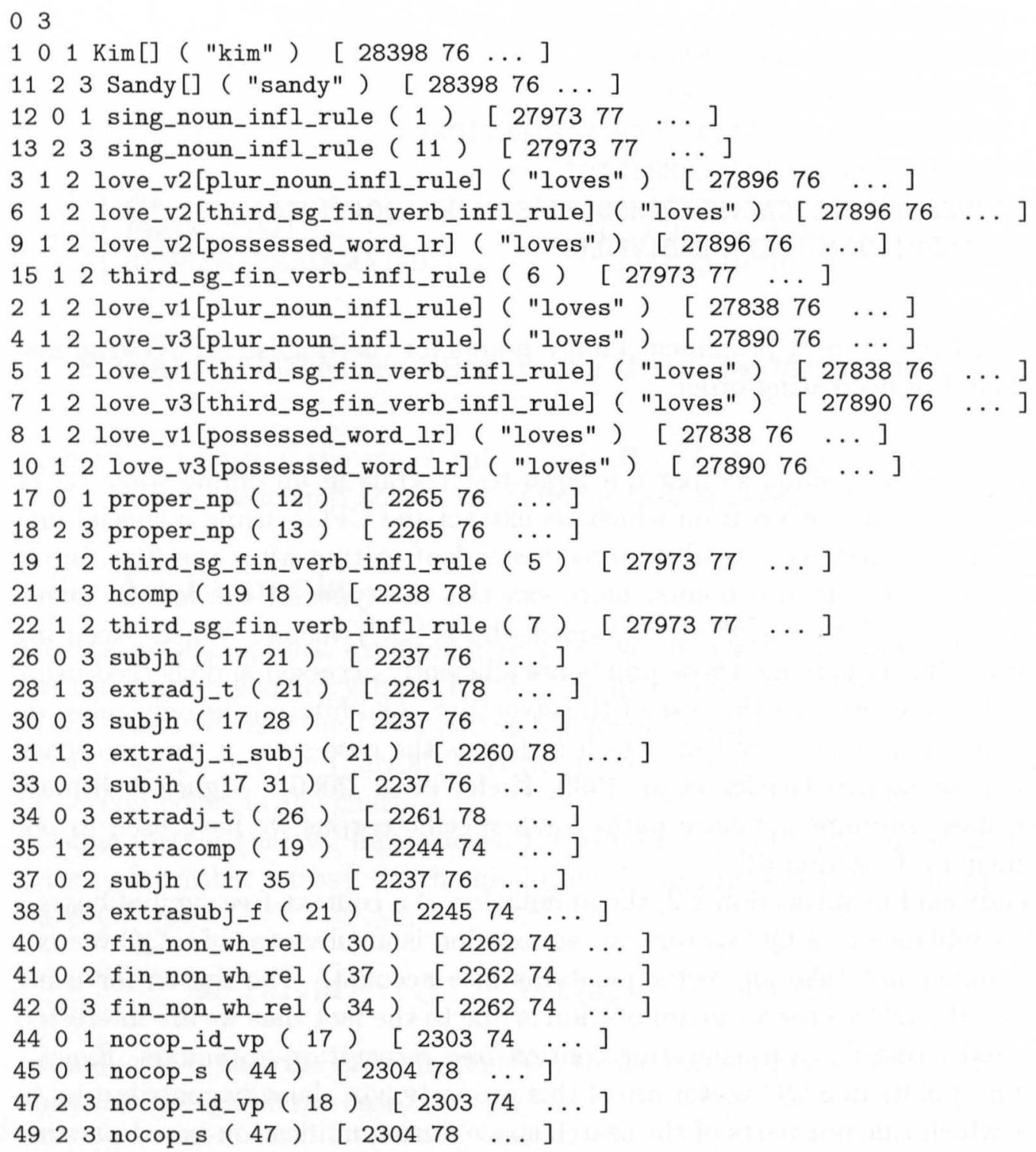

Figure 2: Parts of the chart for the sentence Kim loves Sandy. Note that the chart is topologically sorted, i.e., it is guaranteed that edge ids are introduced before they are referred to in the immediate daughters list. For instance, edge 13 refers to edge 11 (i.e., originating from a unary rule), edge 30 to edges 17 and 28 (a binary rule), etc. The edges 1-11 can be thought of as realizing the lexicon lookup. Notice that loves is assigned nine readings (different senses \& morphosyntactic variations). The unary rules $12,13,15,19$, and 22 are morpholexical rules. The unary rule proper_np $(17,18)$ raises a noun to an NP. Some passive edges $(40-42)$ result in useless $\mathrm{CF}$ rules for this sentence, since they represent dead branches in the search space. 


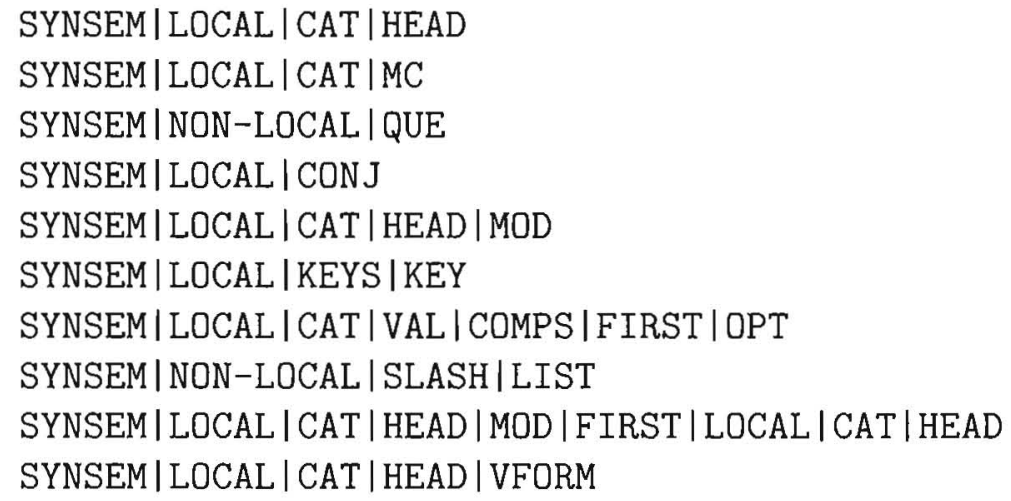

Figure 3: The ten most prominent failure points for the English HPSG grammar (June 2002) in decreasing order.

prominent failure points, we parse a large test corpus in an offline stage (t,f bo precise, we use the corpus from which we extract the CFG), using a special unification engine that records all failures instead of exiting aftor the first failing unification. These failure points, more exactly, the types of the feature struc:tures at these points. constitute the quick-check $(\mathrm{QC})$ vector. When excouting unification during parsing, those points are efficiently accessed and checked using type unification prior to the rest of the structure. QC filtering heavily relies on type unification being very fast, which in fact is the case since it can be cached or even precompiled (Kiefer et al. 1999, Kiefer et al. 2000). Figure 3 displays the ten most prominent failure paths for a specific corpus we have used in our experiments (cf. section 6).

As already said in subsection 2.2, the annotation of a context-free symbol bears a close resemblance to a $\mathrm{QC}$ vector - an annotation is a subvector of a $\mathrm{QC}$ vector., i.e., we might not take all vector positions into account. The reason for using (parts of) the QC vector as an annotation is due to the fact that we are interested in fast and modestly overgenerating context-free recognition grammars. Exactly the failure points in a $\mathrm{QC}$ vector are of this property, viz., heavily contributing to failures which rule out parts of the search space during unification-based parsing. We note here that not every QC path has to be present in every feature structure, since only certain features are appropriate for certain TFSs. Let us give an example. Given the above set of QC paths, it turns out that the original headcomplement rule hcomp is undefined for the ninth QC path. To account for this problem, we use the type $U$ introduced in subsection 2.1 to express such undefinedness. In the concrete syntax, we write *undef* instead. Consequently, we obtained instantiations of the binary head-complement rule schema hcomp (schema 1 in Pollard and Sag 1994, pp. 38) such as

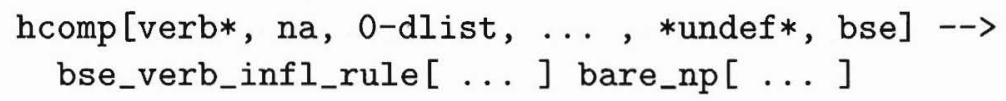

In order to circumvent such undef values, it might be a good idea to work with different annotation vectors for each primary category symbol. This means that 

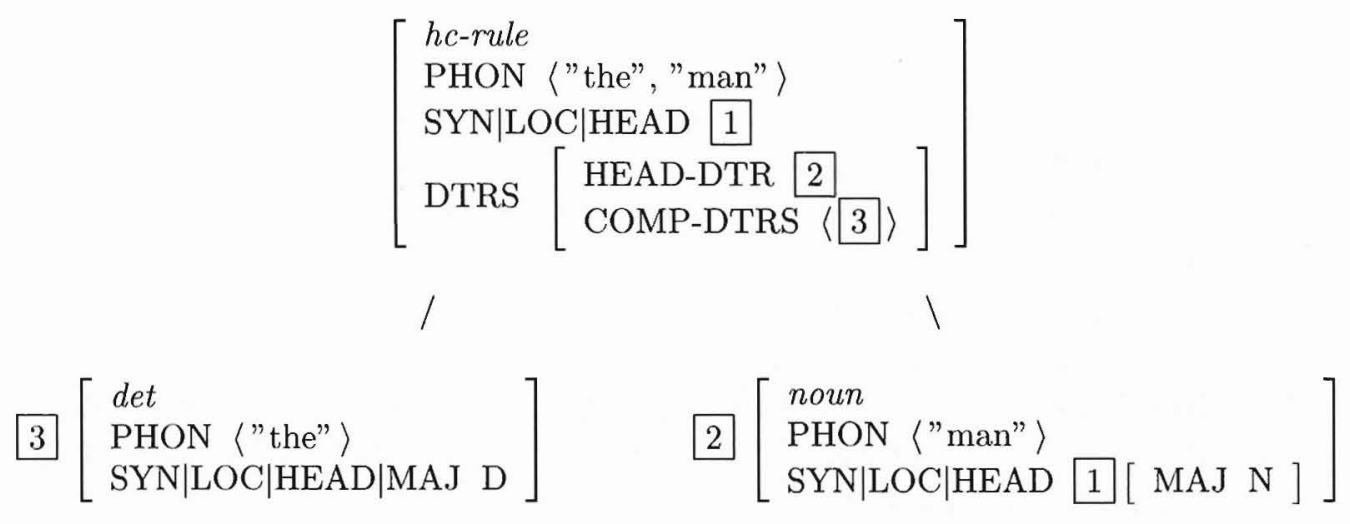

Figure 4: Simplified derivation tree for the phrase the man in HPSG-I.

we have to partition the set of rule names $R=R_{1} \cup \ldots R_{n}$, such that each $R_{i}$ $(1 \leq i \leq n)$ is associated with a set of defined quick-check paths.

\subsection{An Example}

We present a simplified example here to make the approach more clear. We will use the feature geometry from HPSG-I (Pollard and Sag 1987) to make things easier. Assume that the UBG parser has identified the phrase the man, so that it has constructed the (partial) derivation tree in figure 4, which is represented by several edges in the chart.

Assume further that we have chosen the annotation path SYN|LOC|HEAD|MAJ (together with other paths). With this in mind, we can derive the following annotated $\mathrm{CF}$ rule

$$
\mathrm{hc}-\mathrm{rule}[\mathrm{N}, \ldots] \rightarrow \operatorname{det}[\mathrm{D}, \ldots] \operatorname{noun}[\mathrm{N}, \ldots]
$$

assuming that the rule name is identical to the top-level type of the TFS (which must not always be the case).

\subsection{Algorithm}

The idea behind the context-free extraction is relatively straightforward and is given in pseudo code in figure 5.

As we already said, the HPSG parser produces for each input sentence an output file that contains an external representation of the passive edges of the chart for this sentence, encoded in the format given by figure 1. The extraction HPSG2CFG then works as follows. Given a directory D and a vector of quick check paths $Q$, we iterate over the files in $D$ (line 3). For each file, we then construct a vector edges of internal edges (i.e., JAVA objects) for the set of external passive edges stored in this file, using makeEdges (line 4). This includes the in-memory reconstruction of the TFSs for the mother structures (the LHSs). For each vector position, i.e., for each edge e, we build up a LHS symbol (i.e., a pair, see section 2.2), consisting of a name field (via getName) and an annotation 


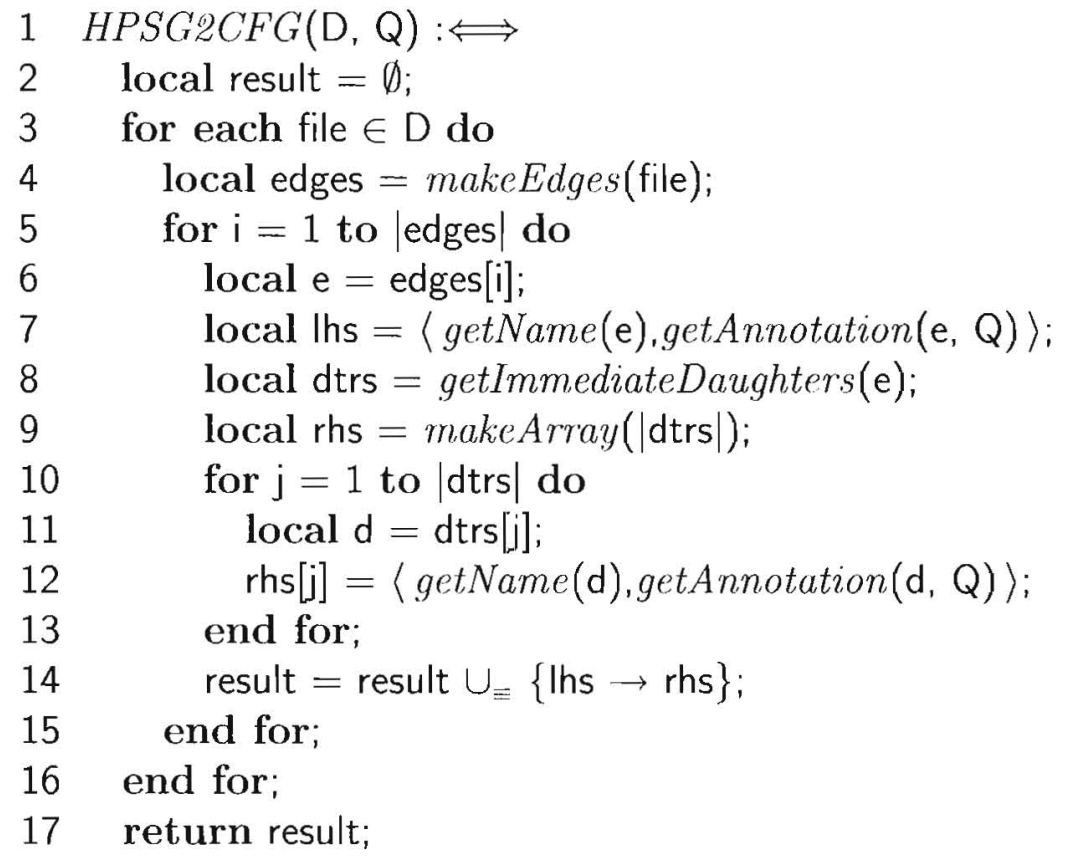

Figure 5: The overall structure of the extraction algorithm.

vector (via getAnnotation), given the quick-check paths $Q$ (line 7). The same is done for every RHS symbol, but since we usually have more than one RHS symbol, we collect them in an array dtrs of length equal to the number of the immediate daughters of the passive chart edge (lines 8 13). For every passive edge, we finally generate a context-free rule object (sce section 2.3), given the LHS and the RHS (line 14). The new CF rules are adjoined to the result set (line 14). After we have processed all files in directory $D$, the result sct is returned at last (line: 17).

The subscript $\equiv$ of the union operator in line 14 of the algorithm should indicate that new rules are added to the result set using rule equivalence. I.e., a new rule only contributes to the final CFG if no structural cquivalent rule has already been introduced earlier during the extraction process. Even for a small corpus, a large number of structural equivalent rules are generated, resulting cither from reappearing words or from reappearing linguistic constructions. The non-astonishing observation is that the smaller the annotation gets, the larger the number of equivalent rules becomes. Clearly, by taking more quick-check paths into account, we obtain more specific CF grammars, consisting of more rules. In the next subsection, we will slightly modify line 14 , replacing the rule equivalence: test by rule subsumption.

\subsection{A Variation}

Rule subsumption, defined in section 2.3, now comes into play to scale down generated grammars. We apply this operation online during the extraction process in that we replace line 14 of the algorithm by

$$
\text { result }=\text { result } \cup_{\preceq}\{\text { lhs } \rightarrow \text { rhs }\} ;
$$


The intention behind $U_{-}$is that a new rule is only added to the result set iff it is more general than at least one rule already in the set. If so, the old rule and porhaps further other rules are removed. If it is more specific, the new rule is clearly not added. The application of that operation guarantees that the rules from the result set result are pairwise incompatible, i.e.,

$$
\forall \alpha, \beta \in \text { result. } \alpha \bowtie \beta \text {, for all } \alpha \neq \beta
$$

$U_{<}$is somewhat related to the specialized union operation $U_{r}$ in Kicfer and Krieger 2002. However, $U_{E}$ operates over typed feature structures representing context-free symbols, whereas our operation is directly applied to annotated CF rules.

Of course, $U_{\prec}$ does change the context-free language $\mathcal{L}_{\prec}$ when compared to the language $\mathcal{L}_{\Sigma}$, resulting from the application of $\cup_{\vdots}$ :

$$
\mathcal{L}_{\equiv} \subseteq \mathcal{L}_{\prec}
$$

Given the number of rules for the two grammars $\mathcal{G}_{\prec}$ and $\mathcal{G}_{-}$, we have

$$
\left|\mathcal{G}_{\unlhd}\right| \leq\left|\mathcal{G}_{\equiv}\right|
$$

A simple example clearly shows this. Assume that the temporary result set contains the following three CF rules

$$
\text { result }=\left\{\begin{array}{l}
\alpha=A \rightarrow \ldots B_{1} \ldots \\
\beta_{1}=B_{1} \rightarrow \ldots \\
\beta_{2}=B_{2} \rightarrow \ldots \\
\ldots
\end{array}\right\}
$$

and assume that the new rule

$$
\beta=B \rightarrow \ldots
$$

subsumes both $\beta_{1}$ and $\beta_{2}$. $\cup_{\preceq}$ would thus delete $\beta_{1}$ and $\beta_{2}$ from the result set and will add $\beta$ to it. Furthermore, since $B_{1}$ and $B_{2}$ are no longer valid ( $\beta_{1}$ and $\beta_{2}$ have been deleted!), we must replace every occurrence of $B_{1}$ and $B_{2}$ by the new nonterminal $B$, introduced in production $\beta$. This, however, has the effect that at least the modified rule $\left(\alpha^{\prime}=A \rightarrow \ldots B \ldots\right)$, derived from $\alpha$, overgenerates.

Our approach keeps track of such rule deletions by implementing a symbol subsumption maintenance graph. In the above example, we establish two associations between $B_{1}, B_{2}$ and $B$ : $\left(B_{1} \mapsto B\right),\left(B_{2} \mapsto B\right)$. Given the example, it is possible that the new rule $\beta$ might even be deleted by a newer, more general rule $B^{\prime} \rightarrow \ldots$ later. In this case, we have to further specify a new association: $\left(B \mapsto B^{\prime}\right)$. In the end, such substitution chains will be dereferenced, so that we can immediately substitute a dead RHS symbol by its correct and existing LHS counterpart. In the example, for instance, we must then know that $B_{1}$ should not be substituted by $B$, but instead by $B^{\prime}$.

We also have to make associations for the converse case - if a new special rule $\gamma$ is not added due to an existing more general rule $\gamma^{\prime}$, we must record this fact by 
creating the association $\left(L(\gamma) \mapsto L\left(\gamma^{\prime}\right)\right)$, since $L(\gamma)$ might occur on the RHS of final CF rules.

We note here that the substitutions are of course not restricted to the LHS symbols only. Consider the following three toy $\mathrm{CF}$ rules which we might have acquired so far

$$
\text { result }=\left\{\begin{array}{l}
\alpha=\mathrm{N}[\mathrm{sg}, \mathrm{fem}] \rightarrow \text { "Mary" } \\
\beta=\mathrm{NP}[\mathrm{sg}, \mathrm{fem}] \rightarrow \mathrm{N}[\mathrm{sg}, \mathrm{fem}] \\
\gamma=\mathrm{S}[\ldots] \rightarrow \mathrm{NP}[\mathrm{sg}, \mathrm{fem}] \mathrm{VP}[\ldots] \\
\ldots
\end{array}\right\}
$$

and assume that the new, more general rule $\beta^{\prime}$

$$
\beta^{\prime}=\mathrm{NP}[\text { num }, \mathrm{fem}] \rightarrow \mathrm{N}[\text { num }, \text { fem }]
$$

now comes in, substituting $\beta$. The result set then changes to

$$
\text { result }=\left\{\begin{array}{l}
\alpha^{\prime}=\mathrm{N}[\text { num }, \text { fem }] \rightarrow \text { "Mary" } \\
\beta^{\prime}=\mathrm{NP}[\text { num }, \text { fem }] \rightarrow \mathrm{N}[\text { num }, \text { fem }] \\
\gamma^{\prime}=\mathrm{S}[\ldots] \rightarrow \mathrm{NP}[\text { num }, \text { fem }] \operatorname{VP}[\ldots] \\
\ldots
\end{array}\right\}
$$

Since we substitute the dead symbols at the very end of the approximation, the resulting $\mathrm{CFG}$ is clearly not optimal, i.e., not minimal. A proper treatment here would require that we have to update the symbol substitution graph (and potentially perform substitutions) each time a new passive edge is checked against the temporary CF rule set. Since we might process several millions of edges during the approximation of a grammar, we do not apply this technique at the moment. However, two alternative treatments circumvent symbol substitutions.

Unary Rules. The idea here is to couch symbol substitutions in terms of additional unary rules. In the above example, we still delete $\beta$ by $\beta^{\prime}$, do not change $\alpha$ and $\gamma$, but add the following two unary rules:

$$
\begin{aligned}
& \mathrm{N}[\mathrm{num}, \mathrm{fem}] \rightarrow \mathrm{N}[\mathrm{sg}, \mathrm{fem}] \\
& \mathrm{N}[\text { num }, \text { fem }] \rightarrow \mathrm{N}[\mathrm{sg}, \mathrm{fem}]
\end{aligned}
$$

Such rules simply express the fact that num and sg are related in the UBG by type subsumption: sg $\preceq$ num. Goldstein 1988 proposed a similar solution, calling the unary rules unification rules.

Online Symbol Subsumption. Since a context-free parser (usually) employs symbol equality at runtime (and not symbol subsumption or unifiability), $\mathrm{N}$ [num, fem] and $N[s g, f e m]$ are regarded to be incompatible, of course. To recover from this behavior, we can clearly apply symbol subsumption (or unifiability) at runtime. In order not to lose performance, this step heavily relies on type unification being very fast, which is the fact, as it can be precompiled (Kiefer et al. 2000) or cached (Kiefer et al. 1999).

At this point of our investigation, we already note here that favoring rule subsumption in terms of rule equivalence does not have any significant advantage 
(less rules, but not that many), but mostly disadvantages (complex handling of dead symbols and rule frequencies, worst running time of the approximation, overgeneration). Nevertheless, wo have implemented rule subsumption, since UBGs might exist which will take advantage of this operation. In this context, it is worth noting that the closely related operation $\cup_{\sqsubseteq}$ made the grammar approximation in Kiefer and Kricger 2002 finally tractable.

\subsection{Computing Start Productions}

One point in the algorithm is still missing, viz., the generation of start produc:tions. We have decided to employ only a single synthetic start symbol s in our grammars. This symbol has to be fresh, i.e., for all symbols $\langle n, a\rangle$ of the extracted grammar, we demand that $N(\mathrm{~s}) \neq n$. In the implementation, the user must specify a non-empty list of start (or root) types, types which subsume original rule definitions, and thus subsumes potential rule instantiations. These types specify wellformedness conditions that a feature structure must satisfy to be a legal uttcrance (e.g., empty subcategorization list). Now let $T$ be the sct of all start types, $R$ the set of extracted CF rules so far, and s the new top-level start symbol.

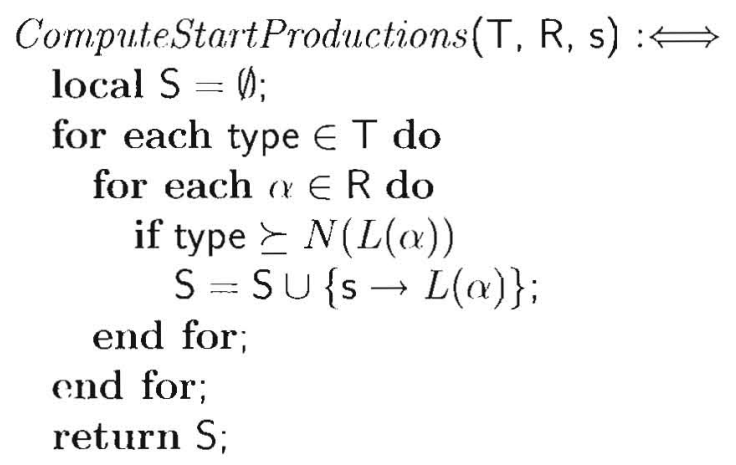

In the English ERG/LinGO HPSG grammar developed at CSLI, sentential phrases are subsumed by the type root_strict, and thus a start symbol in the extracted CFG can be determined by checking whether the name of a LHS symbol is subsumed by this start type. Non-sentential saturated phrases in ERG (e.g., PPs and NPs) are characterized by root_phr, thus if we want find all saturated phrases in the approximated $\mathrm{CFG}$, we have to declare these two types to be start types.

\subsection{Rule Frequency}

As we said in section 2.3, rule objects possess a frequency field which will gain importance if we move from the generated CFG to a trained PCFG which predicts probability distributions over CFG derivations. Exactly the frequency countcr is set to 1 during the initialization of a rule object and is incremented by 1 in case a structural equivalent rule has been detected.

Concerning the frequency field $f$ of a new rule $\alpha$ that has replaced more specific: rules $\alpha_{1}, \ldots, \alpha_{n}$, we have

$$
f(\alpha):=\left(\sum_{i=1}^{n} f\left(\alpha_{i}\right)\right)+1, \text { for all } \alpha_{i} \prec \alpha
$$


since the more general rule now acts as a representative for the deleted specialized rules. Assuming the contrary, the frequency counter of truly general rules $\alpha_{i}$ $(1 \leq i \leq n)$ are incremented by 1 when penalizing $\alpha$ :

$$
f\left(\alpha_{i}\right):=f\left(\alpha_{i}\right)+1, \text { for all } \alpha_{i} \succeq \alpha
$$

Considering the unary start productions from section 3.6, the frequency counter of a start production $s \rightarrow l$ is set to the sum of the frequencies of those rules $\alpha \in R$, whose LHS is exactly $l$, given the set of all CF rules $R$ :

$$
f(s \rightarrow l):=\sum_{\alpha \in R} f(\alpha), \text { where } \alpha=l \rightarrow r_{1} \ldots r_{n}
$$

In order to obtain a proper probability distribution, we have to normalize the rule frequency counter in the standard way. Let $R$ be the set of all extracted CF rules, $f(r)$ be the value of the frequency counter for rule $r \in R$, and $n$ be the total number of all passive edges for a given parsed corpus as delivered by the unification-based parser. We then compute the probability $p(r)$ for a context-free rule $r$ as

$$
p(r):=\frac{f(r)}{n}
$$

which gives us

$$
p(R):=\sum_{r \in R} p(r)=1
$$

\section{Other Approaches}

In this section, we will present several other approaches to compilation and approximation, some of them sound (Pereira and Wright 1991), that is, the language generated by the resulting grammar is a superset of the language of the original grammar, others unsound.

Goldstein 1988 was the first who converted an HPSG to a CFG. Goldstein, however, made several simplified assumptions: (i) English is context-free; (ii) the number of categories/feature structures is finitc; (iii) complex CF (non)terminals are not allowed; (iv) in the early development stage of HPSG, no coreferences and types are used. However, rules are underspecified and lexicalization is alrcady present. This version of HPSG is essentially a modified GPSG. Since Goldstein did not address the (possible) accumulation of information (e.g., under SUBCAT, CONT, or DTRS), a restrictor is clearly not needed. Rule instantiation is rcalized through an active bottom-up parser, using the chart to represent the feature structures.

Carroll 1993 reported in his $\mathrm{PhD}$ thesis on the extraction of CF grammars from unification-based grammars, written in the ANLT (Alvey Natural Language Tools) grammar formalism. Grammars in ANLT usually consist of several hundred of relatively specific GPSGish rules with features having only finitely many values. Categories (= sets of feature-value pairs) can be defined in ANLT, but 
are not explicitly arranged in a type hierarchy. A single ANLT unification rule is mapped one-to-one onto a $\mathrm{CF}$ rule by introducing atomic symbols on the $\mathrm{CF}$ side that abstract from categories in the ANLT grammar. In order to avoid subsumption over CF symbols, subsuming categories are mapped onto a representative that encodes the least upper bound, similar to our operation $\cup_{\preceq}$. A related approach is described in Nakazawa 1995.

A compilation of HPSG, obeying certain restrictions, into lexicalized featurebased TAG is described in Kasper 1992 and Kasper et al. 1995. From an HPSG perspective, this compilation in principle allows a faster parsing system, due to the weaker generative capacity of TAGs (mildly context-sensitive). The idea is to execute parts of HPSG derivations at compile time (viz., the reduction of selection features in selection daughters), producing lexically-anchored feature structures that encode the application of several HPSG rule schemata.

Diagne et al. 1995 and Kasper and Krieger 1996 present a distributed parsing approach that is distinguished by the use of a very restricted HPSG whose derivation trees are reparsed deterministically (in fact, in parallel) by the original HPSG. The two parsers mutually restrict their search space, using a specialized protocol.

Kasper et al. 1996 employ the same idea, but substitute the restricted HPSG through the (relatively specific) context-free backbone of an HPSG-like grammar. By using the pure backbone and a corpus, a PCFG is trained and used online in order to obtain the $n$-best paths of a word lattice. The collection of these paths constitutes the initial chart of the second parser that uses the HPSG essentially for semantic construction.

Neumann and Flickinger 1999 and Neumann 2003 describe an approach that obtains a stochastic lexicalized tree grammar (SLTG) for a given corpus. The idea here is that the training corpus is parsed using an HPSG grammar and an HPSG parser, and derivation trees are iteratively decomposed top-down, resulting in nonterminal nonheaded subtrees, where the cutting points are marked for later substitution. SLTGs are processed by an LTAG-like parser in a two-step process, consisting of an initial all-path parsing phase, followed by the application of the relevant HPSG feature constraints. This idea is related to LFG parsing, but has the clear advantage that a larger tree context is involved (although SLTGs are of context-free power). Since the extraction process of the SLTG grammar works on the derivation trees of a tree bank, viz., tsdb (Oepen and Flickinger 1998), the node labels are relative coarse generalizations of the information embodied in the feature structures used during HPSG parsing. The vagueness or underspecification of the node labels, however, is partly compensated by the larger tree context. The approach furthermore applies two postprocessing techniques: a linguistically-motivated decomposition of trees and a specialization of node labels.

Moore 1999 describes a compilation method that turns unification grammars with finitely-valued features into context-free grammars. The grammar, he reports on, is written in the core language engine formalism of SRI Cambridge, and consists of about 900 relatively specific phrasal rules. Moore only consider finitely-valued features. In order to avoid the combinatorial explosion of rules, Moore only instantiates those features in daughter categories that are constrained by the unification rule and considers only combinations of feature values by uni- 
fying active and passive edges, as in a bottom-up active chart parser. He also throws away useless rules. Moore's paper presents a new left recursion elimination algorithm, specifically tuned for the grammar formalism GSL of the Nuance speech recognizer (Nuance 2004), taking advantage of the fact that GSL allows regular expressions. Moore also consider various combinations of acoustic and symbolic information in a language model and is a great overview paper. In a certain sense, Moore's approach is the forerunner for Rayner et al. and Dowding et al., laying the foundations for the more sophisticated enumerative compilation techniques below. Moore's expansion technique will probably not work for lexicalized theories, such as HPSG.

Kiefer and Krieger 2000, Kiefer and Krieger 2002, and Kiefer and Krieger 2004 present a sound approximation method that turns unification-based grammars, such as HPSG or PATR-II into context-free grammars. The method does not rely on a corpus, but is purely grammar-driven. In an initial phase, the method generalizes the set of all lexicon entries, by abstracting from word-specific information. The abstraction is specified by means of a restrictor. After that, the grammar rules are instantiated by unification, using the abstracted lexicon entries and resulting in derivation trees of depth 1. A rule restrictor is applied to each resulting feature structure, removing all information contained only in the daughters of a rule. Additionally, the restriction gets rid of information that will either lead to infinite growth of the feature structures or that does not constrain the search space. The restricted feature structures (together with older ones) then serve as the basis for the next instantiation step. Again, this results in TFSs encoding a derivation, and again the TFSs are restricted. The iteration is proceeded until a fixpoint is reached, meaning that further iteration steps will not compute additional information. Given the TFSs from the fixpoint, it is then easy to generate context-free productions, using the full feature structures as symbols of the CFG. The speedup factor for the aged and eng2000 testsuites within a two-stage parsing architecture are between 1.7-2.7.

In Kiefer et al. 2002, an extension of the method is presented which easily allows the disambiguation of UBG readings, by indirectly relying on a trained PCFG, derived from the approximated CFG. Considering a random baseline of $72 \%$ for the exact match task, the method shows an increase of $16 \%$ ( $=88 \%$ precision).

In Cancedda and Samuelsson 2000, a corpus-based specialization method is introduced which directly operates on rules written in the LFG framework. Because the LFG formalism allows RHSs of grammar rules to consist of regular expressions (REs), the idea of this framework is to expand RHSs into RE-free sequences of symbols, guided by the training data. Since Kleene star and complementation in REs as well as specialized operators like shuffle may introduce spurious ambiguities, such simplified rule instantiations clearly speed up parsing (up to a factor 6). The downside of this method is that one might lose coverage (about 13\%). To compensate for the loss in coverage, a two-stage parsing architecture is proposed in which a second stage, consisting of the original grammar, is only invoked in case the first specialized parser failed. Even with this backup mechanism, a speedup between 1.8-2.7 was obtained.

Dowding et al. 2001 compares the approach to grammar approximation in Moore 
1999 to that in Kiefer and Krieger 2000. As a basis for the comparison, they choose a command-and-control grammar written in the Gemini/CLF formalism (we use the same grammar in section 6.4). The motivation for this cnterprise comes from the use of the resulting CFG as a context-free language model for the Nuance speech recognizer (Nuance 2004). The measurements in Dowding et al. 2001 differ from those later conducted in Kiefer and Krieger 2002 and Kiefer and Krieger 2004. Unfortunately, it is not clear why Dowding's implementation of Kiefer \& Krieger's method comes off so badly. Dowding report on an average ambiguity per sentence of 15.4 , whercas Kiefer \& Krieger comes up with only 1.49 (ambiguity rate for the original grammar was 1.44). The compilation time to obtain the approximated grammars differs too: 11 minutes vs. 34 seconds. With a slightly different sctting, Kiefer \& Krieger even obtain a correct approximation, showing that the UBG is in fact of only context-free power. The paper by Dowding et al. 2001 also explores techniques for transforming CFGs into woakly equivalent grammars with less ambiguity. These investigations are important since the use of linguistically-motivated CFGs as language models often lead to confidential paths in the language model labeled with the same recognition hypothesis, so that other good hypotheses are forced out of the beam of the recognizer.

Rayner et al. 2000 conduct a serics of experiments that employ approximated CFGs as language models in the Nuance speech recognizer. Instead of using a domain-specific UBG, Rayner et al. 2000 start with a general, linguisticallymotivated grammar, but use a domain-specific lexicon, resulting in a domainspecific CFG. Compiling a UBG is done by enumerating all possible instantiations of features in rules. To make this approach tractable, a relatively complex mechanism is implemented to arrive at features, having only finitely-many values, similar to the approach in Moore 1999. A kind of rule folding is also applied here (see also section 5.2). The compilation failed for a mid-to-large-size UBG, so they started with a small grammars and incrementally add further rules and lexicon entries. It turned out that a more complex clause structure was not responsible for the poor recognition performance of the resulting CFGs, but instead a small number of rules, basically centered around relative clause modification. The findings in this detailed paper might also be of interest to our method. In Rayner et al. 2001a, the explicit assumption is made that each UBG feature has a finite range of possible values. How this can be guaranteed is not explained in the paper. Further technical aspects of the above compilation method are elaborated. In the related paper Rayner et al. 2001b, it is argued that agreement constraints from the UBG should be kept in the CFG to obtain better language models.

Bos 2002 comes up with a compilation method that is very related to that of Moore and Rayner et al. above. Again, features only have finitely many values and complex values are not allowed. What makes his approach unique, however, is that he shows how to transfer compositional semantics from the UBG into the CFG, using the grammar specification language GSL from the Nuance recognizer package. GSL supports slot filling for constructing semantically-relevant output, similar to W3C's VoiceXML or SRGS, and Sun's JSGF. The challenge for saving the semantics on the CFG side is elimination of left recursion in the rules and 
no support for features and unification. In the end, deriving a logical form for a string is achieved by running the CF parser on that string, using the approximated CFG, followed by several $\beta$-reduction steps. Recognition performance in terms of speed and word error rate is not affected by his method. Bos' way to achieve a compositional semantics is quite close to our proposed treatment of semantic construction, using additional semantic rules (see section 5.5).

\section{$5 \quad$ Summary and Extensions}

As already explained in the paper, the corpus-driven approximation method is unsound, that is, given a corpus $C$ of training sentences and a set of annotation paths $A$ (from which we determine the annotation of a context-free symbol), the approximated CF language is usually not a superset (but also not a subset) of the language accepted by the HPSG (see figure 6). This is due to the fact that not all linguistic constructions licensed by the HPSG are covered by the training corpus, but also because not every piece of information from the TFS is encoded in the annotation of a CF symbol:

$$
\forall C, A \cdot \mathcal{L}(\mathrm{HPSG}) \not \subset \mathcal{L}(\mathrm{CFG}, C, A)
$$

It is easy to see that more training samples $C^{\prime}$ result in a broader language:

$$
\forall C^{\prime}, C \subseteq C^{\prime} . \mathcal{L}(\mathrm{CFG}, C, A) \subseteq \mathcal{L}\left(\mathrm{CFG}, C^{\prime}, A\right)
$$

The subset relation turns around with more annotations:

$$
\forall A^{\prime}, A \subseteq A^{\prime} . \mathcal{L}(\mathrm{CFG}, C, A) \supseteq \mathcal{L}\left(\mathrm{CFG}, C, A^{\prime}\right)
$$

In both cases, more training sentences and/or more annotation paths result in larger rule set. Overall, we can say that the more information from the feature structure is put into the annotations of the context-free symbols, the better the CFG approaches the HPSG in terms of the number of readings and the less it overgenerates w.r.t. linguistic constructions contained in the training corpus. Remember, annotated CF rules approximate HPSG (passive) chart edges, and the existence of more specific CF symbols and rules helps to better mimic the behavior of the HPSG during CF parsing. Finding the right annotation paths, of course, depends on the application domain in which the extracted CFG is employed.

The correlation between UBGs and the family of approximated CFGs w.r.t. a given corpus and a set of annotation paths is depicted in figure 6 .

As motivated in section 3.5, when fixing a corpus $C$ and a set of annotation paths $A$, the language obtained under $U_{\equiv}$ is always a subset of the language resulting from the application of $\cup_{\preceq}$, given the same HPSG source grammar:

$$
\mathcal{L}_{\equiv}(C, A) \subseteq \mathcal{L}_{\preceq}(C, A)
$$

However, the more general grammar has less rules due to the fact that $\cup_{\preceq}$ might delete more than one specialized rule when favoring a more general rule. 


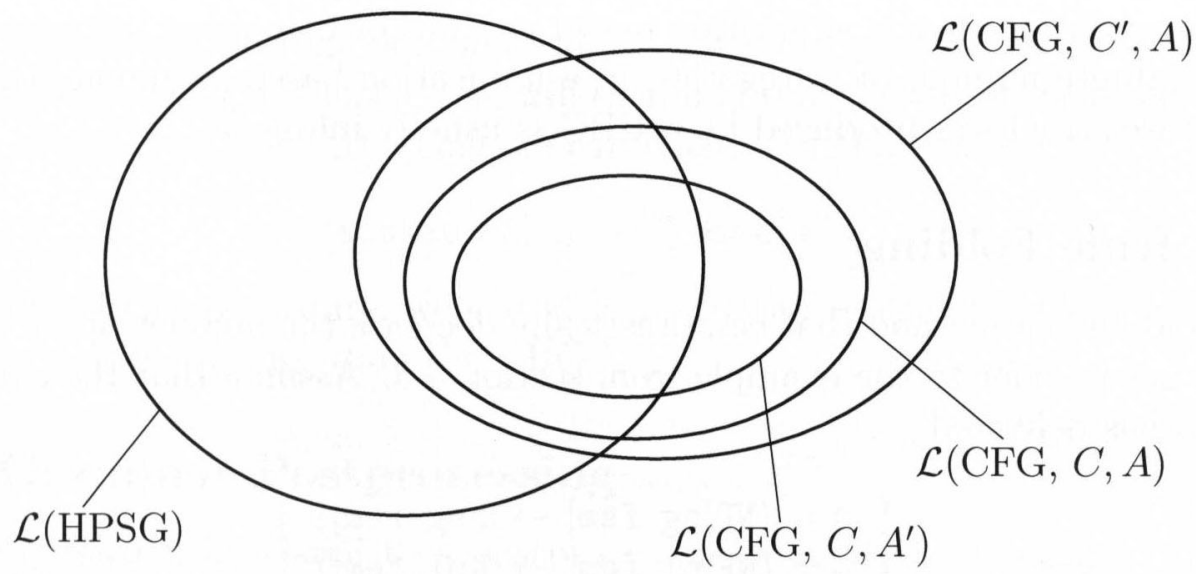

Figure 6: The correlation between the language accepted by the HPSG $\mathcal{L}$ (HPSG) and the approximated context-free language $\mathcal{L}(\mathrm{CFG}, C, A)$, given a corpus $C$ and a set of annotation paths $A$. The approximated language $\mathcal{L}\left(\mathrm{CFG}, C^{\prime}, A\right)$ monotonically grows when given a larger corpus $C^{\prime} \supseteq C$. The language $\mathcal{L}(\mathrm{CFG}$, $C, A^{\prime}$ ) usually shrinks when given a larger set of annotation paths $A^{\prime} \supseteq A$ (but the number of rules grows).

We explained in the beginning that we have taken all passive edges of the HPSG parser into account - this is a compromise. Two extremes obviously stand out: (1) using only passive edges which have led to complete HPSG readings and (2) taking all, i.e., active and passive edges into account. Approach 1 clearly generates a smaller, but more approaching language when compared to the language of the HPSG source grammar. Approach 2 leads to a much broader language due to the fact that active edges contain uninstantiated daughter positions which are usually not much restricted by the rule schemata in HPSG.

Another avenue which is worth to follow results from widening our domain of locality by moving from context-free rules to trees of depth greater than 1 . This is not hard to achieve since the transmitted chart of the HPSG parser not only allows us to refer to the immediate daughters of an edge, but even to every daughter covered by this edge. Our open framework encourages experimentation towards this direction.

We will now discuss several postprocessing steps which might be applied after a CFG has been extracted.

\subsection{Elimination of Useless Rules}

Since the extracted annotated grammars are still context-free, we can remove those symbols which will never contribute to a reading. These symbols and the productions using them are called useless and there exist a fast decidable algorithm that, given a grammar, produces a smaller grammar which does not contain useless productions (Hopcroft and Ullman 1979, pp. 87). The runtime efficiency of a CF parser clearly benefits from the removal of such useless productions, helping the parser to avoid dead derivation branches. Note that, in general, unsuccessful 
rule instantiations in the unification parser can not be computed beforehand, due to the infinite number of 'categories' in a unification-based grammar (the set of all feature structures produced by a UBG is usually infinite).

\section{$5.2 \quad$ Rule Folding}

Rule folding is a method that can drastically decrease the number of CF productions. Let us refer to the example from section 2.3. Assume that the extraction process has delivered

$$
\left\{\begin{array}{l}
\alpha=(\mathrm{NP}[\mathrm{sg}, \mathrm{fem}] \rightarrow \mathrm{N}[\mathrm{sg}, \mathrm{fem}]) \\
\beta=(\mathrm{NP}[\mathrm{pl}, \mathrm{fem}] \rightarrow \mathrm{N}[\mathrm{pl}, \mathrm{fem}])
\end{array}\right\}
$$

Rule folding will then replace $\alpha$ and $\beta$ by

$$
\gamma=(\mathrm{NP}[\text { num }, \mathrm{fem}] \rightarrow \mathrm{N}[\text { num }, \mathrm{fem}])
$$

since $\gamma$ covers exactly the two variations of the number feature, viz., singular and plural. Because NP $[\mathrm{sg}, \mathrm{fem}], \mathrm{NP}[\mathrm{pl}, \mathrm{fem}], \mathrm{N}[\mathrm{sg}, \mathrm{fem}]$, and $\mathrm{N}[\mathrm{pl}, \mathrm{fem}]$ are now dead symbols, we must again replace their occurrence in every rule by $\mathrm{NP}[$ num, fem $]$ and $\mathrm{N}[\mathrm{num}, \mathrm{fem}]$, resp.

Rule folding $F$, of course, does change the language induced by the original extracted CFG $\mathcal{G}$ and produces a more general grammar. The reason for this goes along the argument presented at the end of section 3.5.

$$
\mathcal{L}(\mathcal{G}) \subseteq \mathcal{L}(F(\mathcal{G}))
$$

\subsection{Automatic Lexicon Extension}

New utterances not covered by the corpus are only recognized by the extracted CFG if they can be mapped to already parsed syntactic constructions and to already seen words. One can imagine that the relevant domain-specific syntactic constructions can be covered by a sufficiently large training corpus. Lexical gaps, however, should not be attacked by taking more samples into account. One way to enlarge the CF lexicon is by mapping lexicon entries from the HPSG to related terminal symbols in the $\mathrm{CFG}$, originating from parsing the training corpus. Assume, the training corpus contains

\section{Bill gives Mary a book}

but does not mention the word cookie. A sentence such as

\section{Bill gives Mary a cookie}

will then not be recognized.

The extraction process has represented book by the unary production

$$
\text { book_n1 }[\ldots] \rightarrow \text { "book" }
$$


where book_n1 can be seen as a preterminal, speaking in terms of CFGs.

By inspecting the HPSG lexicon, we will then find that book is of the same lexical type as cookic, vi\%, bookn1. Thus we can cnlarge the CF lexicon by

$$
\text { cookie_n1 }[\ldots] \rightarrow \text { "cookie" }
$$

By exploiting this procedure, we can at least guarantee that the CFG will have the same lexical coverage than the UBG.

\subsection{Grammar Postprocessing}

Lexicon extension is related to a postprocessing step which "massages" the approximated CFGs, more precisely, the rules which have a preterminal symbol such as in [prep*, ...] on their RHS. For instance, we might have

$$
\begin{aligned}
& \text { in }[\text { prep*, ...] } \rightarrow \text { "- in" } \\
& \text { hcomp[prep*, ...] } \rightarrow \text { in[prep*, ...] proper_np[noun_or_nomger, ...] }
\end{aligned}
$$

Now assume that our training corpus does not contain the preposition on and that automatic lexicon extension has added the following "lexicon lookup" (or lexical) rule

$$
\text { on }[\text { prep*, ...] --> "on" }
$$

However, we are still missing the very likely $\mathrm{CF}$ rule

$$
\text { hcomp [prep*, ...] } \rightarrow \text { on [prep*, ...] proper_np[noun_or_nomger, ...] }
$$

Constructing such new rules should, however, not that difficult. Clearly, when addressing these topics properly, the need for a larger training corpus is not that demanding.

\subsection{Constructing Meaningful Output}

In case we are not only interested in a context-free boolean recognizer, but would like to see some useful output (e.g., MRS-like structures; see Copestake et al. 2001), we can either apply two-stage parsing here (see above), letting the UBG deterministically replay the $\mathrm{CF}$ parse trees, or (automatically) equip the approximated CFGs by 'semantic rules', similar to attribute grammars, a framework extensively used in syntax-directed translation, dating back to the early sixties (Aho et al. 1986). ${ }^{5}$ The latter approach completely obviates UBG parsing and has the further advantage that overgeneration within the extracted CFG will not be prohibited by a subsequent, more restrictive UBG reparse.

Such semantic rules can not be obtained directly from the passive edges of a chart (or even from the extracted CF rules), but instead must be reconstructed from the CF rules with the help of the HPSG rule schemata, together with the total surjective mapping from CF rules to HPSG rule schemata.

Let us give an example. Assume that the training corpus contains the sentence

\footnotetext{
${ }^{5}$ Such semantic rules nowadays gain importance in the speech grammar community, most notably in the Semantic Interpretation for Speech Recognition (Van Tichelen 2003) framework for the Speech Recognition Grammar Specification (Hunt and McGlashan 2004).
} 
for which we obtain, among other rules,

$$
\operatorname{subjh}[\operatorname{verb}, \ldots] \rightarrow \text { proper_np}[\text { noun, ...] verb_infl_rule[verb, ... }]
$$

The unique (slightly simplified) HPSG rule schema associated with the LHS of subjh $[$ verb, ...] plus the inherited HPSG principles gives us a feature structure which exactly specifies the 'transport' of information from the daughters to the mother $(\oplus$ denotes list append):

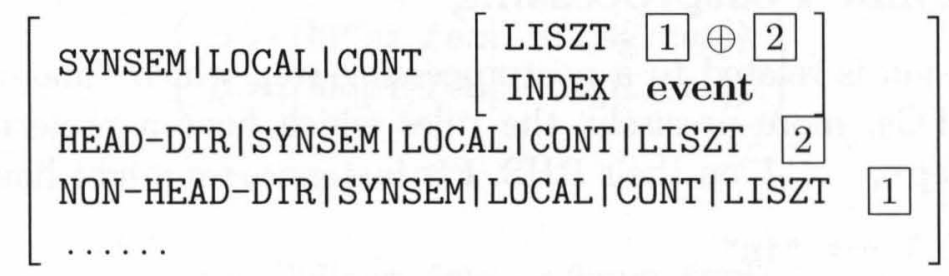

This transport is expressed through coreferences (1 and 2 $)$ and has to be remodeled in the semantic rules attached to the $\mathrm{CF}$ rule. We thus enrich the above extracted CF production by the following two simplified semantic rules (we use a kind of PATR-II notation, where 0 denotes the LHS, 1 the first RHS daughter, etc.):

$$
\begin{aligned}
& \text { O.SEM.LISZT }:=1 . \text { SEM.LISZT } \oplus \text { 2.SEM.LISZT } \\
& \text { 0.SEM.INDEX }:=\text { event }
\end{aligned}
$$

The process of semantic composition must be grounded in the lexicon, thus we equip each lexicon entry of the CFG by the relevant semantic rules which can be obtained from the corresponding lexicon entries of the HPSG (let $\langle\ldots\rangle$ denotes a list of elements):

$$
\begin{aligned}
& \text { Tigger }[\ldots] \rightarrow \text { "Tigger" } \\
& \text { O.SEM.LISZT }:=\langle\text { Tigger }\rangle
\end{aligned}
$$

But perhaps the much simpler procedure of annotating CF symbols with additional, semantically-relevant information from the feature structures might suffice. In this case, successful CF derivation trees will be reinspected in a second phase, where the according semantic information is reconstructed. This approach could also be the starting point for approximating not only recognition grammars, but even useful generation grammars.

\section{Experiments}

We have applied our method to five different grammars at this point of writing. We took these grammars from Kiefer and Krieger 2002. The first three grammars are small-size UBGs, used primarily for showing interesting properties of the approximation method. The fourth grammar is an HPSG-like encoding of John Dowding's mid-size unification grammar, written in the Gemini/CLE formalism. The fifth grammar is the large English Resource Grammar, developed at CSLI, Stanford. 


\subsection{A Grammar for $a^{n} b^{n}$}

The first binary-branching grammar licenses the language $\left\{a^{n} b^{n} \mid n>0\right\}$ and is shown together with the two lexicon entries for $a$ and $b$.

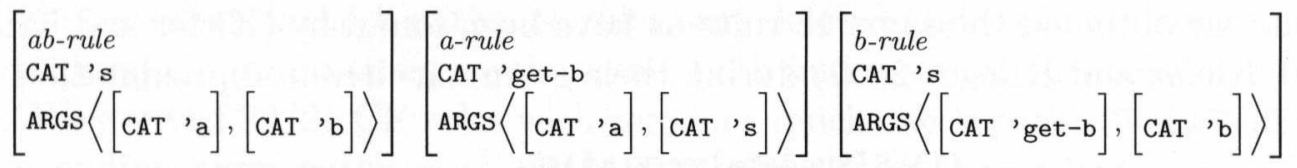

$$
\begin{aligned}
& {\left[\begin{array}{ll}
a & \\
\text { CAT } & , a
\end{array}\right]\left[\begin{array}{ll}
b & \\
\text { CAT } & , b
\end{array}\right]}
\end{aligned}
$$

The obvious quick-check path here is CAT. Both ab-rule and b-rule are start symbols (category 's) which lead to the introduction of two unary start productions. The below rules are exactly of the form generated by the extraction process and are identical with the rules generated by the purely grammar-driven approximation in (Kiefer and Krieger 2000, Kiefer and Krieger 2002).

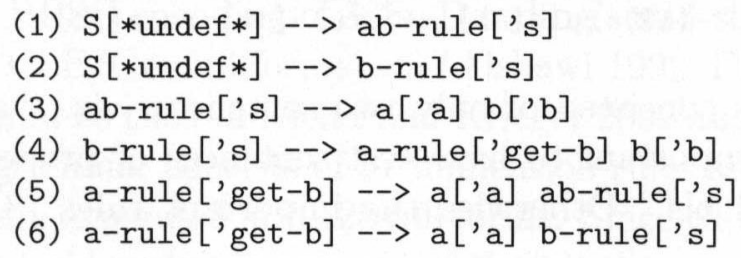

The training corpus consisted of three sentences, viz., "a b", "a a b b", and "a $\mathrm{a} a \mathrm{~b}$ b b". Note that the generated CF language is in fact a correct approximation, i.e., it derives exactly the same language as described by the unificationbased grammar. Even the single sentence "a a a b b b" would suffice to reach this grammar. Note further that we can generate the same annotated CFG by specifying only a single non-wellformed sentences, e.g., "a a a b b". In order to compute the full set of the above six rules, it is important to have at least a training sentence that starts with three as, otherwise rule (6) is not derivable. This rule is responsible for the infinite nature of $\left\{a^{n} b^{n} \mid n>0\right\}$.

Speaking in terms of generative power, it is obvious that the resulting grammar is not optimally small: there is a weakly equivalent grammar with three instead of six productions. If the distinction between the different rules (rule types) in the nonterminals is dropped, the remaining symbols are [*undef $*],\left[{ }^{\prime} \mathrm{a}\right],\left[{ }^{\prime} \mathrm{b}\right]$, ['s], and ['get-b], hence allowing us to collapse productions (1) and (2), as well as (5) and (6). In addition, the resulting unary production [*undef*] --> ['s] could also be deleted, assuming ['s] now to be the start symbol.

\subsection{A Grammar With Coreferences}

Our second example employs coreference constraints in an extremely ambiguous grammar to show the variation of the CAT feature between the mother and the two daughters in the annotated CF rules. Note that the dynamic behavior of coreferences in an UBG at runtime can not be modeled in a CFG, i.e., the distinction between type and token identity. Only instantiations of coreference constraints in an UBG at a certain point of time are in the reach of descriptive means of CFGs. 


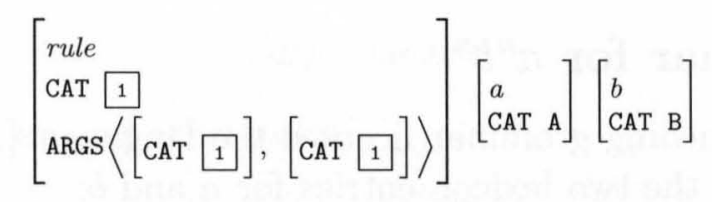

Again, we obtained the same 12 rules as have been found by (Kiefer and Krieger 2000, Kiefer and Krieger 2002) during their grammar-driven approximation.
(1) $\mathrm{S}$ [*undef*] $\rightarrow \mathrm{a}[$ 'a]
(2) $\mathrm{S}$ [*undef*] $\rightarrow \mathrm{b}\left[\mathrm{\prime}^{\mathrm{b}}\right]$
(3) $\mathrm{S}[*$ undef $*] \rightarrow \operatorname{rule}\left[{ }^{\prime} \mathrm{a}\right]$
(4) $\mathrm{S}\left[*\right.$ undef*] $\rightarrow$ rule $\left[{ }^{\prime} \mathrm{b}\right]$
(5) rule['a] $\rightarrow a[$ 'a] a ['a]
(6) $r u l e\left[{ }^{\prime} a\right] \rightarrow a\left[{ }^{\prime} a\right]$ rule['a]
(7) rule['a] $\rightarrow$ rule['a] a['a]
(8) rule['a] $\rightarrow$ rule['a] rule['a]
(9) rule ['b] $\rightarrow b\left[{ }^{\prime} b\right] b[' b]$
(10) rule $\left[{ }^{\prime} b\right] \rightarrow b\left[{ }^{\prime} b\right]$ rule $\left[{ }^{\prime} b\right]$
(11) $\operatorname{rule}[' \mathrm{~b}] \rightarrow \operatorname{rule}\left[{ }^{\prime} \mathrm{b}\right] \mathrm{b}\left[{ }^{\prime} \mathrm{b}\right]$
(12) $\operatorname{rule}\left[{ }^{\prime} b\right] \rightarrow \operatorname{rule}[' b]$ rule ['b]

Our training corpus consisted of only two sentences, viz., "a a a a" and "b b b b". Again it is important to have test sentences of proper length (viz., four consecutive a's and b's. Otherwise the important rules (8) and (12) are not derivable.

\subsection{Shieber's PATR-II Grammar}

The third example is the feature structure encoding of Shieber's second sample PATR-II grammar (Shieber 1986, pp. 71-76). This grammar uses two underspecified rules for verb phrase construction as in Uther persuades knights to storm Cornwall. It is clearly a test case much more in the direction of UBGs than the first two examples. Overall, the grammar consists of three rules.

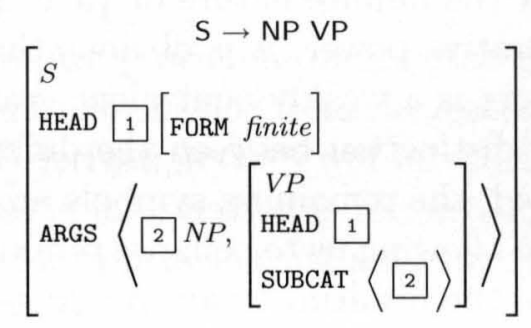

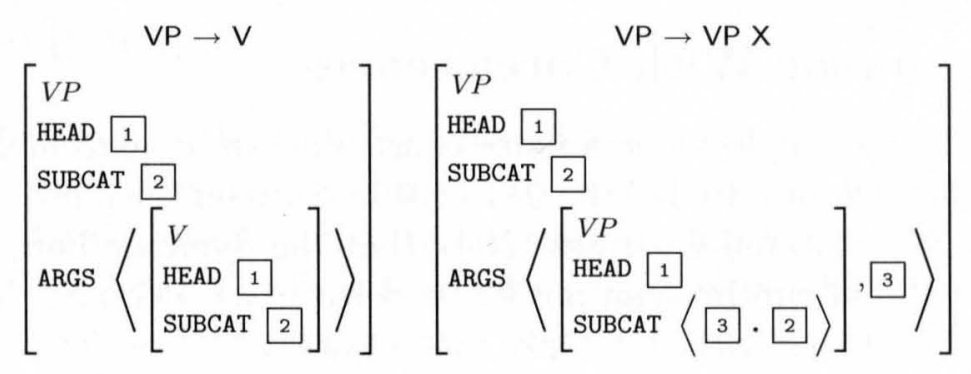


together with 13 lexicon entries.

We made several tests, varying the number of quick-check paths, both under equivalence and subsumption. First of all, we note that independent of the kind and number of quick-check paths, no rules were deleted under rule subsumptionthe resulting $\mathrm{CF}$ grammars were the same, both under equivalence and subsumption. We derived 20/21 CF rules with zero/one quick check path (SUBCAT|REST). When adding more paths, e.g., HEAD|FORM, the CF grammar had a maximum number of 25 rules. Additional quick check paths had no further effects.

We have chosen a training corpus of 12 positive and negative sentences. Overall, we obtained the same rules as reported in Kiefer and Krieger 2000 and Kiefer and Krieger 2002). However, the extracted grammars do not contain any useless rules (which was the case for Kiefer \& Krieger).

\subsection{Dowding's Gemini Grammar}

This grammar is an HPSG encoding of John Dowding's mid-size unification grammar, written in the CLE/Gemini formalism (Alshawi 1992, Dowding et al. 1993). It is the same grammar as used in Kiefer and Krieger 2002 and Kiefer and Krieger 2004. The Gemini grammar consists of 57 unification rules and a small lexicon of 216 entries which were expanded into 425 full forms. Since the grammar allows for atomic disjunctions (and makes heavy use of them), the construction of disjunctive normal form resulted in 1,886 type definitions overall. ${ }^{6}$ We used a training corpus of 500 sentences that transforms into 401-528 CF rules, depending on the number of annotation paths $(0,2,3,5,10,17,21)$.

The table in figure 7 gives a summary of the quality of the approximated CFGs under rule equivalence, when compared to the original UBG. In order to measure the degree of CFG overgeneration, we used the same corpus here. When taking the best 21 quick-check paths of the UBG into account, we obtain a perfectly fitting grammar, resulting in a speedup by a factor of more than 82 . More QC path, of course, do not have further effects on the number of readings. The UBG and the CFG measurements were obtained with the help of the bottom-up active chart parser of the PAGE system (Uszkoreit et al. 1994). Since the parser is not optimized for context-free parsing, a larger performance gain is likely to be obtained. ${ }^{7}$ The same parser was also used during the measurements concerning the English HPSG (see next section).

We next measured the number of passive edges and the number of readings for the CFGs that were approximated under rule subsumption (figure 8. Note that the family of grammars below contain useless rules. For these slightly more general grammars, we obtained approximately the same number of passive edges and more important, the same readings. Since the rule sets for these approximated grammars are so small and nearly of the same size, running time for the more

\footnotetext{
${ }^{6}$ Thanks to Mark-Jan Nederhof for implementing the Gemini-to- $\mathcal{T D} \mathcal{L}$ converter.

${ }^{7}$ The measurements were conducted on an $833 \mathrm{MHz}$ Pentium III machine under Linux and Allegro Common Lisp 6.2. Gap introduction was switched off in the PAGE parser in this setting here due to the fact that PET (who produces the input charts for the approximation; cf. section 3.1) can not ground such rules in epsilon items, and hence we will not see such information in the CFG.
} 


\begin{tabular}{|l||r|r|r|r|r|r|}
\hline & \#rules & \#ppedges & \#readings & runtime[s] & overgeneration & speedup \\
\hline \hline UBG & 57 & 19,716 & 666 & 6.59 & 1.00 & 1.00 \\
\hline CFG-0e & 401 & 24,815 & 4,028 & 0.23 & 6.05 & 28.65 \\
CFG-2e & 401 & 24,662 & 1,376 & 0.20 & 2.07 & 32.95 \\
CFG-3e & 454 & 23,364 & 982 & 0.16 & 1.47 & 41.19 \\
CFG-5e & 455 & 23,379 & 982 & 0.15 & 1.47 & 43.93 \\
CFG-10e & 505 & 21,327 & 975 & 0.11 & 1.46 & 54.92 \\
CFG-17e & 525 & 21,007 & 819 & 0.10 & 1.23 & 65.90 \\
CFG-21e & 528 & 20,672 & 666 & 0.08 & $\mathbf{1 . 0 0}$ & $\mathbf{8 2 . 3 8}$ \\
\hline
\end{tabular}

Figure 7: Measurements for the family of approximated grammars obtained under rule equivalence. \#ppedges abbreviates the number of packed passive edges. Overgeneration was measured against the UBG.

general grammars was on par with the more specialized ones. The replacement of specialized symbols in favor of more general ones started when we moved to at least three symbol annotations. A maximum number of 71 final symbol substitutions (\#substitutions) were made for these three annotations (CFG-3s). The maximum number of 16 associations (\#associations) between symbols and their more general replacements (see section 3.5) were obtained for ten annotations (CFG-10s).

\begin{tabular}{|l||r|r|r|r|r|r|}
\hline & \#rules & \#associations & \#substitutions & \#useless & \#ppedgess & \#rearlings \\
\hline \hline CFG-0s & 401 & 0 & 0 & 20 & 24,815 & 1,028 \\
CFG-2s & 401 & 0 & 0 & 43 & 24,662 & 1,376 \\
CFG-3s & 429 & 11 & 71 & 43 & 23,485 & 982 \\
CFG-5s & 432 & 9 & 51 & 13 & 23,480 & 982 \\
CFG-10s & 472 & 16 & 52 & 71 & 21,431 & 975 \\
CFG-17s & 495 & 14 & 43 & 86 & 20,992 & 819 \\
CFG-21s & 498 & 14 & 43 & 86 & 20,695 & 666 \\
\hline
\end{tabular}

Figure 8: Measurements for the family of approximated grammars obtained under rulc subsumption. \#useless abbreviates the number of useless rules.

Compared to the results reported in Kiefer and Krieger 2002, it is not astounding that our grammars are much smaller, since they are derived from a corpus, and not merely by the UBG alone. Of course, the approximated CFGs in Kiefer and Krieger 2002 do cover a greater variety of linguistic constructions.

We also measured the time of two-stage parsing (total time of CF parsing plus UBG replay) against the UBG baseline $(5.7 \mathrm{sec})$ for the grammars CFG-10e and CFG-21e. We obtained a speedup factor between 9.2 (=0.62 sec) and 10.2 (= $0.56 \mathrm{sec})$. The next section shows that the speedup is getting larger when taking more complex structure as well as longer sentences into account. 


\subsection{CSLI's English Resource Grammar (LinGO)}

Wo also applied our method to the large English LinGO grammar (June 2002), developed at CSLI Stanford. The grammar consists of 61 rule schemata, 8,082 non-leaf types, and a lexicon of 6,930 stems.

\subsection{1 aged}

Wo used the aged test suite (Oepen and Callmeier 2000), consisting of 96 syntactically highly diverse sentences in order to measure the quality of our approximation (average sentence length: 8.4; maximal length: 19). aged consists of 202 stems that cover a great deal of morphological and lexical variation. 719 full forms were computed from these stems. The I'E'I parser which produced the imput charts of passive edges (section 3.1) ran under no restrictions and computed 267,651 passive edges overall. One of the sentence from aged even contributed 139,028 edges. Approximation was performed under rule equivalence and rule subsumption. The length of the annotation vector was varied between 0 and 32 .

Figure 9 shows the asymptotic behavior for the number of nonterminal and rules, when compared to the length of the annotation (sce also figure 3). The explosion of rules from five to six annotations is due to the path SYNSEM|LOCAL |KEYS |KEY who potentially results in more than 4,000 possible values, representing relativcly word-specific information. It is a good idea not to use such path values as annotations in case the rule set gets too large. Furthermore, approximated CF rules will get too specific when incorporating such information. An optional strategy to cope with such a value overload is described in Kiefer and Krieger 2002 and Kiefer and Krieger 2004, viz., type generalization.

The next two tables present runtime measurement and show that the useless rules outweigh when moving to larger annotation vectors. The numbers also show (at least for aged and csli, see next section) that the grammars obtained under rule equivalence are nearly of the same size as those obtained under rule subsumption, but are overall better when regarding the number of readings (\#readings). This will clearly gain importance in the second stage of an UBG-replay approach. Considering the UBG parser, 1,589 readings for aged were found. PET failed for 17 of the 96 sentences when using the English HPSG.

A plot of the number of rules obtained under rule equivalence against the number of samples is shown in figure 12 .

We also conducted measurements for two grammars under rule equivalence. The pure HPSG parser, fully equipped with quick-check filtering, resulted in an overall parse time of 1,361.5 seconds. Total time of two-stage parsing (CF parsing plus UBG replay using the full HPSG) for aged was

- 110 annotations: $59.4 \mathrm{sec}=22.9 \times$ speedup

- 226 annotations: $52.8 \mathrm{sec}=25.8 \times$ speedup

Considering runtime, the grammar with 226 annotations was better, since HPSG replay had needed less unifications. 


\begin{tabular}{|r||r|r|r|r|}
\hline \#annotations & \#E-rules & \#S-rules & \#associations & \#substitutions \\
\hline \hline 0 & 3,475 & 3,475 & 0 & 0 \\
1 & 3,783 & 3,539 & 28 & 264 \\
2 & 3,890 & 3,552 & 52 & 1,210 \\
3 & 4,047 & 3,579 & 71 & 1,539 \\
4 & 4,048 & 3,580 & 71 & 1,536 \\
$\mathbf{5}$ & 4,141 & 3,670 & 69 & 1,510 \\
$\mathbf{6}$ & 8,585 & 7,406 & 733 & 4,547 \\
7 & 8,662 & 7,574 & 605 & 3,895 \\
8 & 9,430 & 7,998 & 760 & 4,096 \\
9 & 10,050 & 8,459 & 891 & 3,969 \\
10 & 11,364 & 9,335 & 1,189 & 4,032 \\
11 & 11,469 & 9,341 & 1,200 & 4,199 \\
12 & 11,532 & 9,641 & 1,079 & 3,624 \\
13 & 11,591 & 9,667 & 1,087 & 3,670 \\
14 & 11,771 & 9,704 & 1,123 & 3,856 \\
15 & 11,857 & 9,800 & 1,120 & 3,896 \\
16 & 11,909 & 9,829 & 1,111 & 3,921 \\
17 & 11,928 & 9,830 & 1,123 & 3,954 \\
18 & 11,928 & 9,850 & 1,107 & 3,931 \\
19 & 12,127 & 9,913 & 1,183 & 4,057 \\
20 & 12,169 & 9,926 & 1,201 & 4,050 \\
25 & 13,182 & 10,867 & 1,295 & 4,020 \\
32 & 15,173 & 12,389 & 1,454 & 4,014 \\
\hline
\end{tabular}

Figure 9: Total number of rules for the aged test suite, obtained under both equivalence (E-rules) and subsumption (S-rules). The explosion in the number of rules from 5 to 6 is due to the path SYNSEMILOCAL|KEYS|KEY.

\begin{tabular}{|r||r|r|r|r|r|r|}
\hline \#annotations & \#useful & \#useless & \#ppedges & \#readings & \#errors & runtime $[\mathrm{s}]$ \\
\hline \hline 0 & 3,453 & 22 & 122,311 & $4.294 E+29$ & 2 & 3.09 \\
2 & 3,737 & 153 & 73,010 & $6.527 E+18$ & 3 & 0.72 \\
5 & 3,943 & 198 & 64,364 & $1.408 E+17$ & 4 & 0.54 \\
10 & 7,160 & 4,204 & 23,607 & 617,068 & 9 & 0.13 \\
18 & 7,291 & 4,637 & 21,970 & 307,483 & 9 & 0.12 \\
32 & 8,236 & 6,937 & 22,858 & 174,533 & 9 & 0.10 \\
110 & 10,237 & 15,455 & 19,440 & 11,614 & 15 & 0.09 \\
226 & 13,682 & 32,025 & 18,855 & 11,195 & 15 & 0.09 \\
\hline
\end{tabular}

Figure 10: Useful and useless rules for aged, obtained under rule equivalence (without lexicon lookup rules).

\subsection{2 csli}

The csli test suite which originated from the old HP test suite developed at Hewlett Packard Labs (Oepen and Flickinger 1998), consists of 5,720 grammatical and ungrammatical samples. It is intended to cover a great deal of the syntactical constructions of English. Average sentence length is 5.0, maximal length 20. 585 full forms (= \#CF terminal symbols), e.g., "little", and 3269 lexicon lookup 


\begin{tabular}{|r||r|r|r|r|r|r|}
\hline \#annotations & \#useful & \#useless & \#ppedges & \#readings & \#errors & runtime[s] \\
\hline \hline 0 & 3,453 & 22 & 122,311 & $4.294 E+29$ & 2 & 3.09 \\
2 & 3,418 & 134 & 68,027 & $3.644 e+19$ & 3 & 0.69 \\
5 & 3,475 & 195 & 64,878 & $2.303 E+18$ & 4 & 0.65 \\
10 & 7,022 & 2,313 & 31,509 & $3,202,651$ & 9 & 0.14 \\
18 & 7,080 & 2,770 & 25,369 & $1,617,231$ & 9 & 0.13 \\
32 & 7,391 & 4,998 & 23,569 & 326,149 & 9 & 0.13 \\
110 & 8,919 & 12,694 & 19,347 & 17,004 & 15 & 0.09 \\
226 & 12,824 & 30,205 & 18,262 & 13,253 & 15 & 0.09 \\
\hline
\end{tabular}

Figure 11: Useful and useless rules for aged, obtained under rule subsumption (without lexicon lookup rules).

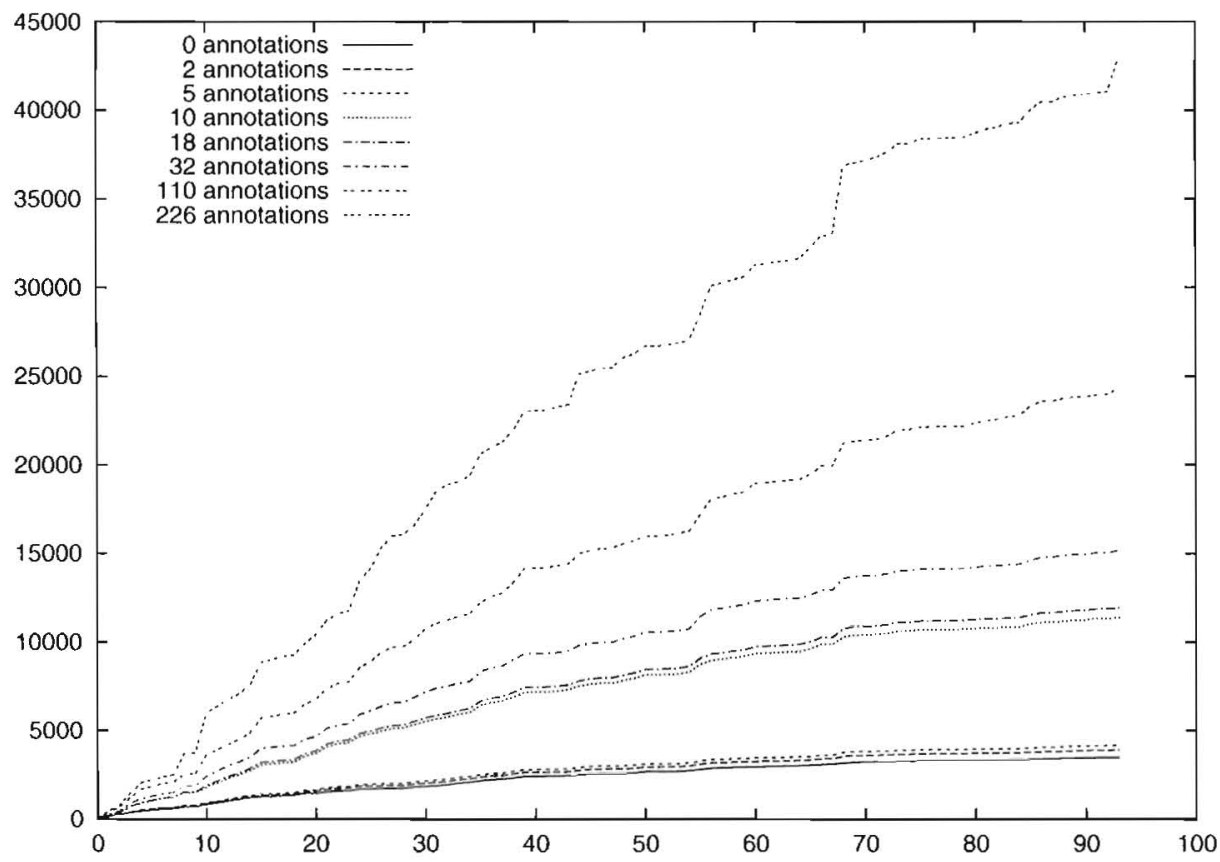

Figure 12: Plot of the number of rules obtained under rule equivalence against the number of samples from aged. Note that we depict the sum of useful and useless rules without start productions here. The table clearly shows that, at least for 226 symbol annotations, the training corpus is too small.

rules (= \#preterminal symbols or lexical categories), e.g.,

$$
\text { little_det[det, na, 0-dlist, ...] --> "little" }
$$

can be found in every approximated grammar (i.e., average ambiguity rate per stem $\approx 5.6$ ). The maximal number of passive chart edges in the PET parser was set to 100,000 . 7,981 readings were found by PET.

For csli, we obtained the following numbers for the approximated CFGs (see figure 13 and 14).

Some of the numbers from figure 14 were astounding on first sight. Firstly, the decrease of useful rules from 10 to 18 annotations seems to contradict to what has 


\begin{tabular}{|r||r|r|r|r|}
\hline \#annotations & \#E-rules & \#E-nonterms & \#S-rules & \#S-nonterms \\
\hline \hline 0 & 11,432 & 3,372 & 11,432 & 3,372 \\
2 & 12,845 & 3,561 & 11,736 & 3,473 \\
5 & 14,007 & 3,796 & 12,252 & 3,637 \\
10 & 52,848 & 16,508 & 41,530 & 11,056 \\
18 & 54,376 & 17,212 & 43,203 & 11,623 \\
32 & 65,808 & 24,373 & 54,875 & 17,940 \\
\hline
\end{tabular}

Figure 13: Number of rules (including start productions and lexicon lookup rules) and nonterminals for the csli test suite, obtained both under equivalence (E-rules, E-nonterms) and subsumption (S-rules, S-nonterms). The rule set both contains useful and useless rules.

\begin{tabular}{|r||r|r|r|r|r|r|}
\hline \#annotations & \#useful & \#useless & \#ppedges & \#readings & \#errors & runtime[s] \\
\hline \hline 0 & 8,161 & 2 & $2,981,080$ & $6.492 E+41$ & 356 & 86.12 \\
2 & 9,138 & 438 & $2,116,619$ & $3.026 E+32$ & 510 & 40.22 \\
5 & 10,059 & 679 & $1,752,303$ & $2.464 E+25$ & 627 & 26.02 \\
10 & $\mathbf{3 2 , 3 6 6}$ & 17,383 & 589,711 & $241,665,239$ & 1,744 & 4.66 \\
18 & $\mathbf{3 1}, \mathbf{5 7 5}$ & 19,702 & 545,788 & $56,629,513$ & 1,898 & 4.63 \\
32 & 32,354 & 30,489 & 534,236 & $38,030,870$ & 1,920 & 4.20 \\
110 & 40,742 & 79,520 & 436,342 & $\mathbf{1 8 , 2 6 6}$ & $\mathbf{2 , 9 2 6}$ & 4.08 \\
226 & 58,394 & 187,755 & 377,187 & $\mathbf{2 2 , 4 9 7}$ & $\mathbf{3 , 0 6 5}$ & 4.12 \\
\hline
\end{tabular}

Figure 14: Useful and useless rules for csli, obtained under rule equivalence (without lexicon "lookup" rules). Less than a fourth of all rules are useful under the most specific CFG. Note the decrease of useful rules when moving from 10 to 18 annotations, even the total number of rules increases. Note also the increase of readings from 110 to 226 annotations, even though the recognized language gets smaller (cf. number of errors). Overgeneration against the UBG w.r.t. training corpus was between 2.29 and 2.82 for the two largest grammars.

already been said before. However, we have always argued that the total number of all rules increases when moving to a larger annotation. This decrease is due to the fact that "older" useful rules have been outdated, i.e., have become useless through the specialization of some of their CF symbols (more annotations).

Secondly, the number of readings might increase when we equip the CF symbols with more information. Again, this does not stand in contrast to what has already been said. Clearly, a more specialized CF grammar (226 annotations) recognizes a smaller language than a more general CFG (110 annotations). This fact is supported by the number of errors in figure 14 (more errors). "Older" CF rules, here, are split up into new specialized instances when adding more annotations. In a certain sense, new information might add spurious ambiguities. Thus, the $\mathrm{CFG}$ with 110 annotations can be seen to better approximate the UBG than 1 he bigger CFG. This behavior should be taken into consideration in the context of two-stage UBG replay parsing. 


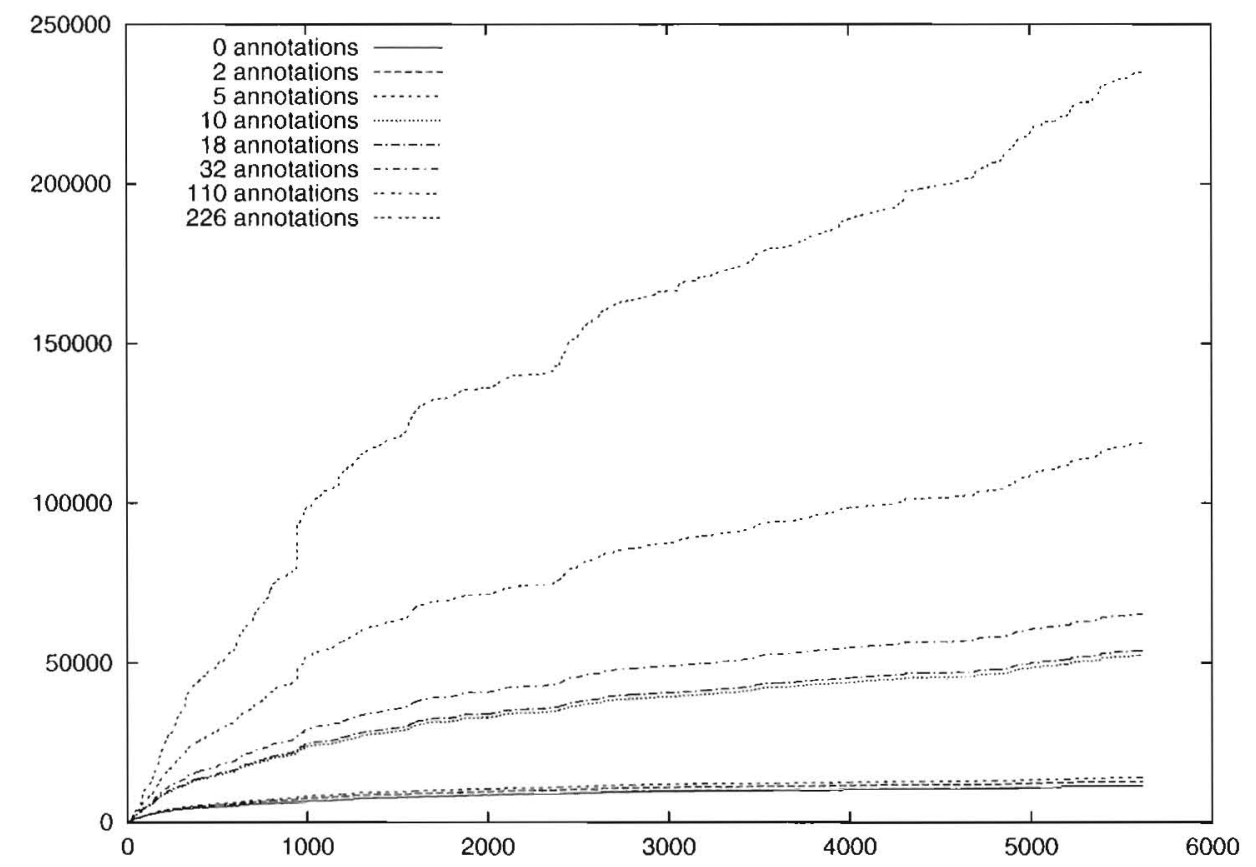

Figure 15: Plot of the number of rules obtained under rule equivalence against the number of samples for csli. Note that we depict the sum of useful and useless rules without start productions here. Again, the table clearly shows that, at least for 226 symbol annotations, the training corpus is too small.

However, the measurements for two-stage parsing (110 and 226 annotations) arc of a different kind and show that the restrictedness of the CFG (= number of errors) should be taken into account when judging the practical usefulness of a grammar. The grammar with 226 annotations per symbol leads to fewer unification in the TIBG replay stage. HPSG baseline for the 5,720 sentences was 2,777.3 seconds (again, quick-check filtering was switched on).

- 110 annotations: $30.7 \mathrm{sec}=90.5 \times$ speedup

- 226 annotations: $28.5 \mathrm{sec}=97.4 \times$ speedup

This speedup of nearly two magnitudes shows the enormous potential of our method. We finish this section with a plot of the number of rules obtained under rule equivalence against the number of samples (figure 15).

\section{Conclusion and Outlook}

In this report, we have described a corpus-driven method for extracting domainspecific context-free grammars. We have presented a variation of this method that will usually yield smaller grammars while having the drawback of being more general. We have also indicated that the approximated CFGs can be turned into PCFGs for disambiguating UBGs in a two-step parsing approach. Furthermore, the approximated CFGs are of interest to domain-specific NLP applications which 
are eagerly waiting for cheap and easily-to-produce recognition grammars, e.g., information extraction or language modeling.

The approach neither generates a true superset nor a true subset of the language accepted by the UBG, but clearly better fits the UBG when given a larger training sample and more annotation values. As we have already indicated, this seeming misbehavior is a desired property, when looking for robust domain-specific grammars. The measurements presented here are very encouraging, but needs to be scrutinized in a domain-specific NLP system and perhaps checked against a pure UBG approach.

Several points still need to be worked out in more details. Automatic lexicon extension and grammar postprocessing are important topics in order to let this approach gracefully react to small training samples (see sections 5.3 and 5.4), so that the need for larger training corpora are not that demanding. In case we are not interested in a domain-specific context-free subgrammar of the UBG, even the World Wide Web can be seen as a huge training corpus, due to the following argument. Given a large-scale UBG and a UBG parser (e.g., PET), it is clear that even ungrammatical utterances or utterances not covered by the UBG are worth to be partially parsed, since the UBG parser always comes up with a chart from which we can compute further $\mathrm{CF}$ rules which approximate, at least, legal phrases, although the UBG parser has failed overall. This idea clearly rises or falls with the quality of the UBG. Unknown word not covered by the UBG (mostly named entities) are also an important topic that needs to be addressed. A viable solution here is to have some kind of named entity grammars, processed in a weaker formalism. Such a shallow formalism will then be invoked in a preprocessing phase when unknown words are detected, producing lexicon entries for the UBG, which ultimately will show up in the CFG later. ${ }^{8}$

As indicated in section 5.5, it is worth to extract not only recognition rules, but also to have semantic rules as in attribute grammars, so that our CFGs will come along with some meaningful output. This idea would obviate the need for a second unification-based grammar that replays the CF derivations for the mere purpose of semantic construction. However, a third line can be taken here by employing ideas from Diagne et al. 1995, Kasper and Krieger 1996, and Kasper et al. 1996. Instead of using the full feature structures of a UBG $G$ during replay, we only employ the related grammar $G^{\prime}$ derived from $G$ : rules and lexicon entries in $G^{\prime}$ are exactly the feature structures from $G$ that have been evaporated under an appropriate restrictor $R$ (Shieber 1985): $G^{\prime}=G \backslash R$. Since we are interested in successful UBG derivations and since we like to keep the CF language generalizations in the second replay stage, we delete those constraints on the UBG side which potentially lead to a unification failure (mostly the syntactic constraints). In HPSG-I (Pollard and Sag 1987), specifying the restrictor is easymostly the information under SYN must be deleted. And in case we are interested in a more shallow semantics, some semantic information from SEM has to be deleted too.

\footnotetext{
${ }^{8}$ The shallow processor SProUT (Becker et al. 2002, Krieger et al. 2004) is obviously a good candidate to implement this task, since it is a unification-based formalism and has the ability to read in PET grammars.
} 


\section{References}

Aho, A. V., R. Sethi, and J. D. Ullman. 1986. Compilers: Principles, Techniques, and Tools. Reading, MA: Addison-Wesley.

Ait-Kaci, H. 1986. An Algebraic Semantics Approach to the Effective Resolution of Type Equations. Theoretical Computer Science 45:293 351.

Alshawi, H. (ed.). 1992. The Core Language Engine. ACL-MIT Press Series in Natural Language Processing. MIT Press.

Becker, M., W. Drożdżyński, H.-U. Krieger, J. Piskorski, U. Schäfer, and F. Xu. 2002. SProUT - Shallow Processing with Unification and Typed Feature Structures. In Proceedings of the International Conference on Nutural Language Processing, ICON-2002.

Bos, J. 2002. Compilation of Unification Grammars with Compositional Semantics to Speech Recognition Packages. In Proceedings of the 19th International Conference on Computational Linguistics, COLING 2002, 106 112.

Briscoe, T., and J. Carroll. 1993. Generalized Probabilistic LR Parsing of Natural Language (Corpora) with Unification-Based Grammars. Computational Linguistics 19(1):25-59.

Callmeier, U. 2000. PET-A Platform for Experimentation with Efficient HPSG Processing. Natural Language Engineering 6(1):99- 107.

Callmeier, U. 2001. Efficient Parsing with Large-Scale Unification Grammars. Master's thesis, Universität des Saarlandes.

Cancedda, N., and C. Samuelsson. 2000. Expcriments with Corpus-based LFG Specialization. In Proceedings of the 6th Conference on Applied Natural Language Processing, 204-209.

Carpenter, B. 1992. The Logic of Typed Feature Structures. Tracts in Theoretical Computer Science. Cambridge: Cambridge University Press.

Carroll, J., T. Briscue, and C. Grover. 1991. A Development Environment for Large Natural Language Grammars. Technical Report 233, Computer Laboratory, Cambridge University, UK.

Carroll, J. A. 1993. Practical Unification-based Parsing of Natural Language. PhD thesis, University of Cambridge, Computer Laboratory, Cambridge, England, September.

Copestake, A., A. Lascarides, and D. Flickinger. 2001. An Algebra for Semantic Construction in Constraint-Based Grammars. In Proceedings of the 39th Annual Meeting of the Association for Computational Linguistics, ACL-2001, 132-139.

Diagne, A. K., W. Kasper, and H.-U. Krieger. 1995. Distributed Parsing With HPSG Grammars. In Proceedings of the 4th International Workshop on Parsing Technologies, IWPT'95, 79-86. Also available as DFKI Research Report RR-95-19.

Dowding, J., J. M. Gawron, D. Appelt, J. Bear, L. Cherny, R. Moore, and D. Moran. 1993. GEMINI: A Natural Language System for Spoken-Language Understanding. In Proceedings of the 31st Annual Meeting of the Association for Computational Linguistics, ACL-93, 54- 61. 
Dowding, J., B. A. Hockey, J. M. Gawron, and C. Culy. 2001. Practical Issues in Compiling Typed Unification Grammars for Speech Recognition. In Proceedings of the 39th Annual Meeting of the Association for Computational Linguistics, ACL-2001, $164-171$.

Flanagan, D. 2002. Java in a Nutshell. Beijing: O'Reilly. 4th edition.

Gazdar, G., E. Klein, G. Pullum, and I. Sag. 1985. Generalized Phrase Structure Grammar. Cambridge, MA: Harvard University Press.

Goldstein, S. D. 1988. Using an Active Chart Parser to Convert Any Context Free Grammar to Backus-Naur Form. Master's thesis, Massachusetts Institute of Technology, January.

Hopcroft, J. E., and J. D. Ullman. 1979. Introduction to Automata Theory, Languages, and Computation. Reading, MA: Addison-Wesley.

Hunt, A., and S. McGlashan. 2004. Speech Recognition GrammarSpecification Version 1.0. Technical report, W3C Recommendation 16 March 2004, http://www.w3.org/TR/2004/REC-speech-grammar-20040316/.

Kaplan, R., and J. Bresnan. 1982. Lexical-Functional Grammar: A Formal System for Grammatical Representation. In The Mental Representation of Grammatical Relations, ed. J. Bresnan, 173-281. Cambridge, Mass: MIT Press.

Kasper, R. 1992. Compiling Head-Driven Phrase Structure Grammar into Lexicalized Tree Adjoining Grammar. In Proceedings of the TAG+ Workshop.

Kasper, R., B. Kiefer, K. Netter, and K. Vijay-Shanker. 1995. Compilation of HPSG to TAG. In Proceedings of the 33rd Annual Meeting of the Association for Computational Linguistics, ACL-95, 92-99.

Kasper, W., and H.-U. Krieger. 1996. Modularizing Codescriptive Grammars for Efficient Parsing. In Proceedings of the 16th International Conference on Computational Linguistics, COLING-96, 628-633.

Kasper, W., H.-U. Krieger, J. Spilker, and H. Weber. 1996. From Word Hypotheses to Logical Form: An Efficient Interleaved Approach. In Natural Language Processing and Speech Technology. Results of the 3rd KONVENS Conference, ed. D. Gibbon, 77-88. Berlin: Mouton de Gruyter.

Kiefer, B., and H.-U. Krieger. 2000. A Context-Free Approximation of Head-Driven Phrase Structure Grammar. In Proceedings of the 6th International Workshop on Parsing Technologies, IWPT2000, 135-146.

Kiefer, B., and H.-U. Krieger. 2002. A Context-Free Approximation of Head-Driven Phrase Structure Grammar. In Collaborative Language Engineering. A Case Study in Efficient Grammar-based Processing, ed. S. Oepen, D. Flickinger, J. Tsuji, and H. Uszkoreit, 49-76. CSLI Publications.

Kiefer, B., and H.-U. Krieger. 2004. A Context-Free Superset Approximation of Unification-Based Grammars. In New Developments in Parsing Technology, ed. H. Bunt, J. Carroll, and G. Satta, 229-250. Kluwer Academic Publishers. 
Kiefer, B., H.-U. Krieger, J. Carroll, and R. Malouf. 1999. A Bag of Useful Techniques for Efficient and Robust Parsing. In Proceedings of the 37th Annual Meeting of the Association for Computational Linguistics, ACL-99, 473-480.

Kiefer, B., H.-U. Krieger, and M.-J. Nederhof. 2000. Efficient and Robust Parsing of Word Hypotheses Graphs. In Verbmobil: Foundations of Speech-to-Speech Translation, ed. W. Wahlster, 280 -295. Berlin: Springer.

Kiefer, B., H.-U. Krieger, and D. Prescher. 2002. A Novel Disambiguation Method For Unification-Based Grammars Using Probabilistic Context-Free Approximations. In Proceedings of the 19th International Conference on Computational Linguistics, COLING2002.

Krieger, H.-U. 1995. TDL-A Type Description Language for Constraint-Based Grammars. Foundations, Implementation, and Applications. $\mathrm{PhD}$ thesis, Universität des Saarlandes, Department of Computer Science, September.

Krieger, H.-U. 2001. Greatest Model Semantics for Typed Feature Structures. Grammars 4(2):139-165. Kluwer.

Krieger, H.-U. 2004a. A Corpus-Driven Context-Free Approximation of Hcad-Driven Phrase Structure Grammar. In Proceedings of the International Colloquium on Grammatical Inference, ICGI-2004.

Krieger, H.-U. 2004b. JTFS-A Java Implementation of Typed Feature Structures. Technical memo, DFKI.

Kriegcr, H.-U., W. Drożdżyński, J. Piskorski, U. Schäfer, and F. Xu. 2004. A Bag of Useful Techniques for Unification-Based Finite-State Transducers. In Proceedings of KONVENS 2004, 105 -112.

Krieger, H.-U., and U. Schäfer. 1994. TDL-A Type Description Language for Constraint-Based Grammars. In Proceedings of the 15th International Conference on Computational Linguistics, COLING-94, 893- 899. An enlarged version of this paper is available as DFKI Research Report RR-94-37.

Lari, K., and S. J. Young. 1990. The estimation of stochastic context-free grammars using the inside-outside algorithm. Computer Speech and Language 4:35 56.

Malouf, R., J. Carroll, and A. Copestake. 2000. Efficient feature structure operations without compilation. Natural Language Engineering 6(1):29- 46.

Muore, R. C. 1999. Using Natural-Language Knowledge Sources in Speech Recognition. In Computational Models of Speech Pattern Processing, ed. K. Ponting. Springer.

Nakazawa, T. 1995. Construction of LR Parsing Tables for Grammars Using FeatureBased Syntactic Categories. In Linguistics and Computation, ed. J. Cole, G. Green, and J. Morgan, 199-219. CSLI Lecture Notes.

Nederhof, M.-J. 2000. Practical Experiments with Regular Approximation of ContextFree Languages. Computational Linguistics 26(1):17 -44.

Neumann, G. 2003. Data-driven Approaches to Head-driven Phrase Structure Grammar. In Data-Oriented Parsing, ed. R. Bod, R. Scha, and K. Sima'an. CSLI Publications, University of Chicago Press. 
Neumann, G., and D. Flickinger. 1999. Learning Stochastic Lexicalized Tree Grammars from HPSG. Technical report, German Research Center for Artifical Intelligence (DFKI), Saarbrücken.

Nuance. 2004. Nuance Home http://www. nuance.com.

Oepen, S., and U. Callmeier. 2000. Measure For Measure: Parser Cross-Fertilization. In Proceedings of the 6th International Workshop on Parsing Technologies, IWPT 2000, 183194.

Oepen, S., and D. P. Flickinger. 1998. Towards Systematic Grammar Profiling. Test Suite Technology Ten Years After. Journal of Computer Speech and Language. 12(4):411 436. Special Issue on Evaluation.

Pereira, F. C., and R. N. Wright. 1991. Finite-State Approximation of Phrase Structure Grammars. In Proceedings of the 29th Annual Meeting of the Association for Computational Linguistics, ACL-91, 246 255. An enlarged version is available in: Finite-State Devices for Natural Language Processing, E. Roche \& Y. Schabes (eds.), MIT Press, Cambridge, MA, 1996.

Pollard, C., and I. A. Sag. 1987. Information-Based Syntax and Semantics. Vol. I: Fundamentals. CSLI Lecture Notes, Number 13. Stanford: Center for the Study of Language and Information.

Pollard, C., and I. A. Sag. 1994. Head-Driven Phrase Structure Grammar. Studies in Contemporary Linguistics. Chicago: University of Chicago Press.

Rayner, M., J. Dowding, and B. A. Hockey. 2001a. A Baseline Method for Compiling Typed Unification Grammars into Context Free Language Models. In Proceedings of EUROSPEECH.

Rayner, M., G. Gorrell, B. A. Hockey, J. Dowding, and J. Boye. 2001b. Do CFG-Based Language Models Need Agreement Constraints. In Proceedings of the 2nd Conference of the North American Chapter of the ACL, NAACL2001.

Rayner, M., B. A. Hockey, F. James, E. O. Bratt, S. Goldwater, and J. M. Gawron. 2000. Compiling Language Models from a Linguistically Motivated Unification Grammar. In Proceedings of the 18th International Conference on Computational Linguistics, COLING 2000, 670-676.

Shieber, S., H. Uszkoreit, F. Pereira, J. Robinson, and M. Tyson. 1983. The Formalism and Implementation of PATR-II. In Research on Interactive Acquisition and Use of Knowledge, ed. B. J. Grosz and M. E. Stickel, 39-79. Menlo Park, Cal.: AI Center, SRI International, November.

Shieber, S. M. 1985. Using Restriction to Extend Parsing Algorithms for ComplexFeature-Based Formalisms. In Proceedings of the 23rd Annual Meeting of the Association for Computational Linguistics, ACL-85, 145- 152.

Shieber, S. M. 1986. An Introduction to Unification-Based Approaches to Grammar. CSLI Lecture Notes, Number 4. Stanford: Center for the Study of Language and Information. 
Uszkoreit, H. 1986. Categorial Unification Grammars. In Proceedings of the 11th International Conference on Computational Linguistics, 187194.

Uszkoreit, H., R. Backofen, S. Busemann, A. K. Diagne, E. A. Hinkelman, W. Kasper, B. Kiefer, H.-U. Krieger, K. Netter, G. Neumann, S. Oepen, and S. P. Spackman. 1994. DISCO-An HPSG-based NLP System and its Application for Appointment Scheduling. In Proceedings of COLING-94, 436-440. A version of this paper is available as DFKI Research Report RR-94-38.

Van Tichelen, L. 2003. Semantic Interpretation for Speech Recognition. Technical report, W3C Working Draft 1 April 2003, http://www.w3.org/TR/2003/WD-semanticinterpretation-20030401/.

Zeevat, H., E. Klein, and J. Calder. 1987. Unification Categorial Grammar. In Edinburgh Working Papers in Cognitive Science, 1: Categorial Grammar, Unification Grammar, and Parsing, ed. N. Haddock, E. Klein, and G. Morrill, 195-222. Centre for Cognitive Science, Edinburgh University, UK. 